\title{
Influence of a Moving Mass on the Dynamic Behaviour of Viscoelastically Connected Prismatic Double-Rayleigh Beam System Having Arbitrary End Supports
}

\author{
Jacob Abiodun Gbadeyan' ${ }^{1}$ and Fatai Akangbe Hammed ${ }^{2}$ \\ ${ }^{1}$ Department of Mathematics, University of Ilorin, Ilorin, Kwara State, Nigeria \\ ${ }^{2}$ Department of Mathematical Sciences, Olabisi Onabanjo University, Ago-Iwoye, Nigeria \\ Correspondence should be addressed to Jacob Abiodun Gbadeyan; j.agbadeyan@yahoo.com
}

Received 30 August 2016; Accepted 28 November 2016; Published 26 February 2017

Academic Editor: Maria Bruzón

Copyright ( $\odot 2017$ Jacob Abiodun Gbadeyan and Fatai Akangbe Hammed. This is an open access article distributed under the Creative Commons Attribution License, which permits unrestricted use, distribution, and reproduction in any medium, provided the original work is properly cited.

\begin{abstract}
This paper deals with the lateral vibration of a finite double-Rayleigh beam system having arbitrary classical end conditions and traversed by a concentrated moving mass. The system is made up of two identical parallel uniform Rayleigh beams which are continuously joined together by a viscoelastic Winkler type layer. Of particular interest, however, is the effect of the mass of the moving load on the dynamic response of the system. To this end, a solution technique based on the generalized finite integral transform, modified Struble's method, and differential transform method (DTM) is developed. Numerical examples are given for the purpose of demonstrating the simplicity and efficiency of the technique. The dynamic responses of the system are presented graphically and found to be in good agreement with those previously obtained in the literature for the case of a moving force. The conditions under which the system reaches a state of resonance and the corresponding critical speeds were established. The effects of variations of the ratio $\left(\gamma_{1}\right)$ of the mass of the moving load to the mass of the beam on the dynamic response are presented. The effects of other parameters on the dynamic response of the system are also examined.
\end{abstract}

\section{Introduction}

The problem of determining the dynamic response of elastic structures traversed by moving loads is of significant technological importance and various researchers (Engineers, Physicists, and Applied Mathematicians) continue to pay considerable attention to studying the various corresponding mathematical models [1-14]. Most of these studies have been carried out for simpler structures such as beams, plates, frames, and shells since such elastic structures form the fundamental components of various modern complex structures and the mathematical analysis involved is relatively less complicated. For instance, the trolleys of overhead travelling cranes which move on their girders, as well as bridges on which trains or vehicles move, may be modeled as moving loads on beam [5]. The theory of vibration of single-beam or single-plate system subjected to moving loads with different boundary conditions has been extensively developed with hundreds of articles on it [1-14]. Frýba [2], in particular, gave a comprehensive survey of some of the techniques for solving various versions of this problem. Some engineering applications of the theory of vibration of a single-beam or single-plate system carrying a moving load include the study of the dynamic behaviour of guided circular saws usually used in the wood products industry, modern high-speed precision machinery processes, design of railway bridges, and the machining processes [14]. However, there exist many problems of notable practical significance in many branches of modern industrial, mechanical, aerospace, and civil engineering for which the theory of vibration of single-beam system under a moving load may not hold and hence one has to resort to the vibration theory of double-beam, triple-beam, or multibeam systems traversed by a moving load. Examples of such problems include the vibration of composite materials which is usually modeled using double-beam system. Elastically connected concentric beams are also being used 
as continuous system models for carbon nanotubes and a linear model for interatomic Van der Waals forces is usually provided for by the elastic layers connecting the two beams. As a third example, it is remarked that the coupled behaviour of paper translating with the paper cloth (wire screen) during paper making process is usually studied by modeling the system as two axially translating tensioned beams interconnected by an elastic foundation. Some other significant applications of double-beam system are in (i) passive vibration control, (ii) weight reduction, and (iii) strength and stiffness increase $[15,16]$. It is, nevertheless, observed from literature that, unlike the single-beam system, relatively few works have been carried out for the non-single-beam system carrying moving loads. This is perhaps due to the difficulties encountered in solving the governing coupled partial differential equations. Dublin and Friedrich [17] studied forced vibration of two elastic Euler beams interconnected by spring-damper system. The free vibration and the impact problem of a double-beam system which is made up of identical beams elastically connected were studied theoretically and experimentally by Seelig and Hoppmann [18] and Seelig and Hoppmann II [19], respectively. Kessel [20] studied the excitation of resonance in an elastically connected double beam system by a cyclic moving load while Kessel and Raske [21] carried out the analysis of the dynamic behavior of the system comprising two parallel simply supported beams which were elastically connected and traversed by a cyclic moving load. There exists other interesting studies which have been conducted on double elastic beams [2224]. To the authors knowledge, most of these previous works involving double beams under moving loads are acted upon by only moving forces. In other words, the effect of the inertia of the moving load has not been taken into account. Yet problems involving this effect, though relatively more difficult, are more appropriate representations of the realistic problems usually encountered in practice. As a matter of fact the moving force problem is a special case of the moving mass problem and the difficulty in the latter is due to the singularity appearing in the inertia terms. The solution techniques in most of the above existing works have also been suitable only for simply supported end conditions. However, in recent years many authors paid attention to earthquake resistance systems as well as economic construction. This calls for lighter weight structures. Hence, it becomes necessary to investigate the influence of relatively large masses traversing such structure. The dynamic response of such structures to moving loads whose inertia effect is not negligible should therefore be thoroughly analysed for a rational safe design.

In the present paper, attention is focused on the effect of the mass of a moving load of constant magnitude and velocity on the dynamic response of a finite prismatic double-beam system interconnected by a core. Of particular interest is the influence of the mass of the moving load on the dynamic response of two finite prismatic parallel upper and lower Rayleigh beams connected by a viscoelastic core and having various classical end conditions. This has not been accounted for in previous studies [25]. It is also assumed that the effect of noise is negligible. Hence the influence of either Gaussian or non-Gaussian noise as well as the output constraints [26, 27] is not taken into account. To achieve the desired objective, a general versatile solution technique is developed. This technique is based, in the first instance, on reducing the two governing fourth-order coupled partial differential equations to a set of two second-order ordinary differential equations using generalized finite integral transform. The latter is then simplified using modified Struble's method [1] and solving the resulting set of two coupled ordinary differential equations using a semianalytical method known as differential transform method (DTM). The solution technique is an extended, modified version of the approach developed by the first author (and Oni) in [1] for the dynamic response of (i) a finite Rayleigh beam and (ii) a non-Mindlin rectangular plate under an arbitrary number of concentrated moving masses. The present technique holds for all types of classical end conditions for double-Rayleigh beams acting upon by either moving forces or masses. Its two-dimensional version for double-plate moving load problem can be easily developed. Semianalytical solutions are obtained. The influence of various parameters (especially those of the inertia of the moving load) involved in the problem are presented graphically and discussed qualitatively and quantitatively. The resonance conditions for both the moving force and moving mass problems are also established. Furthermore, the analysis presented is well illustrated using some of the classical end conditions.

The remaining part of this paper is organized as follows: In Section 2, the problem is defined, stating the pertinent governing differential equations as well as the corresponding initial and boundary conditions. The method of analysis is discussed in Section 3 along with the solutions of the moving force and moving mass double-beam problems. Illustrative examples are given in Section 4, followed by the discussion on resonance conditions for the moving force and moving mass double-beam systems in Section 5. Section 6 deals with the numerical analysis of the problem. Finally, concluding remarks are given in Section 7 .

\section{Mathematical Model}

Consider a double-Rayleigh beam system consisting of two finite, prismatic, undamped, parallel upper and lower Rayleigh beams joined together by a viscoelastic layer (core) which is modeled as a set of parallel springs and dashpots as shown in Figure 1. For the sake of brevity and simplicity, the effect of noise on the system is assumed negligible. Thus, the influence of non-Gaussian noises and output constraints $[26,27]$, in particular, on the system is not considered. The upper beam is subjected to a load $P_{1}(x, t)$ having mass $M_{L}$ and moving with a constant velocity $v$. For simplicity, it is assumed that the two beams are identical having the same length $L$, flexural rigidity $E I$, and mass per unit length $\mu$. For convenience the system is, hereby, referred to as system $I$. The dynamic responses $W_{1}(x, t)$ and $W_{2}(x, t)$ of the upper and lower Rayleigh beams, respectively, 


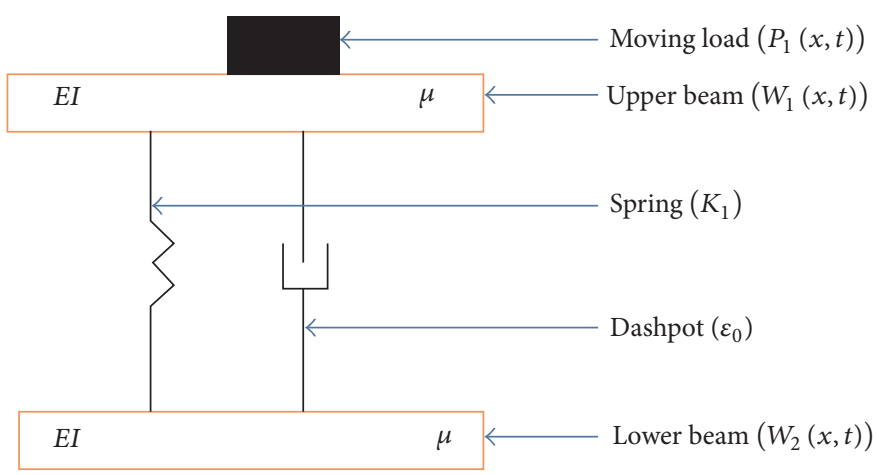

Figure 1: Double-Rayleigh beam system subjected to a moving load $P(x, t)$.

satisfy the following pair of fourth-order, coupled partial differential equations [22, 25].

$$
\begin{aligned}
& E I W_{1}^{i v}(x, t)+\mu \ddot{W}_{1}(x, t)-T_{\text {ra1 }} \mu R_{I} \ddot{W}_{1}^{\prime \prime}(x, t) \\
& \quad+T_{\text {ra } 2}\left[k_{1}\left(W_{1}(x, t)-W_{2}(x, t)\right)\right. \\
& \left.+\varepsilon_{0}\left(\dot{W}_{1}(x, t)-\dot{W}_{2}(x, t)\right)\right]=P_{1}(x, t), \\
& E I W_{2}^{i v}(x, t)+\mu \ddot{W}_{2}(x, t)-T_{\text {ra1 }} \mu R_{I} \ddot{W}_{2}^{\prime \prime}(x, t) \\
& \quad+T_{\text {ra } 2}\left[k_{1}\left(W_{2}(x, t)-W_{1}(x, t)\right)\right. \\
& \left.+\varepsilon_{0}\left(\dot{W}_{2}(x, t)-\dot{W}_{1}(x, t)\right)\right]=0,
\end{aligned}
$$

where $R_{I}$ is the measure of rotatory inertia correction factor, $E$ is the Young's modulus, $I$ is the second moment of area, $k_{1}$ is the spring constant, $\varepsilon_{0}$ is the damping coefficient, $t(0 \leq t \leq$ $L / v)$ is the time, $x$ is the spatial coordinate, the prime denotes differentiation with respect to $x$ and a dot is the differentiation with respect to time $t$, and $T_{\mathrm{ra} 1}$ and $T_{\mathrm{ra} 2}$ are tracing constants, each of which takes on the value unity or zero depending on whether in the subsequent analysis the effects of (i) rotatory inertia and (ii) the joining layer are taken, respectively, into account or not. Furthermore, the concentrated moving load, $P_{1}(x, t)$, is defined as [1]

$$
\begin{aligned}
& P_{1}(x, t)=\left[M_{L} g-T_{\text {ra3 }}\left(M_{L} \ddot{W}_{1}(x, t)-2 M_{L} v \dot{W}_{1}^{\prime}(x, t)\right.\right. \\
& \left.\left.-M_{L} v^{2} W_{1}^{\prime \prime}(x, t)\right)\right][\delta(x-v t)] .
\end{aligned}
$$

In (3) $g$ is the acceleration due to gravity, the term $v^{2} W_{1}^{\prime \prime}(x, t)$ represents the centrifugal acceleration, the term $2 v \dot{W}_{1}^{\prime}(x, t)$ denotes the Coriolis acceleration, the term $\ddot{W}_{1}(x, t)$ represents the local acceleration, and $\delta(\cdot)$ is the Dirac delta function defined as

$$
\delta(x)= \begin{cases}\infty, & x=0 \\ 0, & x \neq 0\end{cases}
$$

and $T_{\mathrm{ra} 3}$ is the third tracing constant whose value is unity if the effect of inertia of the load is taken into account; otherwise, it is zero. Note that the Dirac delta function is an even function; therefore, it is expressed as a Fourier cosine series and we have $[1,3]$

$$
\delta(x-v t)=\frac{1}{L}+\frac{2}{L} \sum_{n=1}^{\infty} \cos \frac{n \pi v t}{L} \cos \frac{n \pi x}{L} .
$$

For the system under consideration, the boundary conditions are any of the classical boundary conditions (i.e., any of the simply supported, free, clamped, and sliding boundary conditions) or their combinations. Hence, the conditions can be written as [1]

$$
\left.B_{1}\left[W_{1}(x, t)\right]\right|_{e}=0=\left.B_{2}\left[W_{2}(x, t)\right]\right|_{e},
$$

where the subscript $e$ indicates that the elements of the vector of linear spatial differential operators $B_{1}$ and $B_{2}$ are to be specified at the boundaries of the two beams. Also, without any loss of generality, it is assumed that the boundary conditions on the same side of the system are the same though they can be any of the classical conditions.

Finally, the initial conditions are

$$
\begin{aligned}
& \left.W_{1}(x, t)\right|_{t=0}=0=\left.\dot{W}_{1}(x, t)\right|_{t=0} ; \\
& \left.W_{2}(x, t)\right|_{t=0}=0=\left.\dot{W}_{2}(x, t)\right|_{t=0} .
\end{aligned}
$$

\section{Method of Solution}

To solve the above initial-boundary-value problem made up of (1)-(3) and (5)-(7), the method of solution already alluded to is presented in this section. The method consists of the following three main steps. (i) Reduce the set of two fourthorder coupled partial differential equations (1) and (2) to a set of two coupled ordinary differential equations of order two using the generalized finite integral transform. (ii) Use the modified asymptotic method of Struble [1] to simplify the resulting set of two coupled transformed ordinary differential equations. (iii) Solve the final set of two simplified coupled transformed ordinary differential equations using a semianalytical method known as differential transform method (DTM). 
3.1. The Transformed Second-Order Coupled Ordinary Differential Equations. To obtain the solution for the transverse dynamic responses $W_{1}(x, t)$ and $W_{2}(x, t)$ of the two Rayleigh beams interconnected by a viscoelastic layer, the fourthorder coupled partial differential equations (1) and (2) are, in the first instance, transformed into a set of two secondorder coupled ordinary differential equations. To this end, the generalized finite integral transforms which are defined as

$$
\begin{aligned}
& \bar{W}_{1}(q, t)=\int_{0}^{L} W_{1}(x, t) U_{q}(x) d x, \\
& \bar{W}_{2}(q, t)=\int_{0}^{L} W_{2}(x, t) U_{q}(x) d x
\end{aligned}
$$

are introduced.

The corresponding inverse formulae are

$$
\begin{aligned}
& W_{1}(x, t)=\sum_{q=1}^{\infty} \frac{\mu}{U_{c q}} \bar{W}_{1}(q, t) U_{q}(x), \\
& W_{2}(x, t)=\sum_{q=1}^{\infty} \frac{\mu}{U_{c q}} \bar{W}_{2}(q, t) U_{q}(x) .
\end{aligned}
$$

In (8) and (9), $U_{q}(x),(q=1,2, \ldots, \infty)$ are eigenfunctions of a single-Euler beam system which usually have the form

$$
\begin{aligned}
U_{q}(x)= & \sin \frac{\lambda_{q}}{L} x+A_{q} \cos \frac{\lambda_{q}}{L} x+B_{q} \sinh \frac{\lambda_{q}}{L} x \\
& +C_{q} \cosh \frac{\lambda_{q}}{L} x .
\end{aligned}
$$

The constants $A_{q}, B_{q}, C_{q}$, and $\lambda_{q}$ are usually determined using any of the classical boundary conditions. Also, the constant $U_{c q}$ is defined as

$$
U_{c q}=\int_{0}^{L} \mu U_{q}^{2}(x) d x
$$

and $U_{q}(x)$ are such that

$$
\operatorname{EIU}_{q}^{i v}(x)=\mu \omega_{q}^{2} U_{q}(x)
$$

while $\omega_{q}$ is the natural circular frequency, defined as

$$
\omega_{q}^{2}=\frac{\lambda_{q}^{4}}{L^{4}} \frac{E I}{\mu}
$$

Taking the generalized finite integral transform of (1)-(3) using (5), (7), (8), (9), (10), and (11), one obtains

$$
\begin{aligned}
& \ddot{\bar{W}}_{1}(q, t)+\omega_{q}^{2} \bar{W}_{1}(q, t)-T_{\mathrm{ra} 1} R_{I} h_{1}^{*}(t)+\frac{T_{\mathrm{ra} 3}}{L} \sum_{q=1}^{\infty} \frac{M_{L}}{\mu U_{c q}} \\
& \cdot\left[h_{3}^{*}(t, v)+2 v h_{4}^{*}(t, v)+v^{2} h_{5}^{*}(t, v)\right] \\
& +T_{\mathrm{ra} 2}\left[\frac{k_{1}}{\mu} \bar{W}_{1}(q, t)+\frac{\varepsilon_{0}}{\mu} \dot{\bar{W}}_{1}(q, t)-\frac{k_{1}}{\mu} \bar{W}_{2}(q, t)\right. \\
& \left.-\frac{\varepsilon_{0}}{\mu} \dot{\bar{W}}_{2}(q, t)\right]=\frac{M_{L}}{\mu} g U_{q}(v t) \\
& \ddot{\bar{W}}_{2}(q, t)+\omega_{q}^{2} \bar{W}_{2}(q, t)-T_{\mathrm{ral}} R_{I} h_{2}^{*}(t) \\
& +T_{\mathrm{ra} 2}\left[\frac{k_{1}}{\mu} \bar{W}_{2}(q, t)+\frac{\varepsilon_{0}}{\mu} \dot{\bar{W}}_{2}(q, t)-\frac{k_{1}}{\mu} \bar{W}_{1}(q, t)\right. \\
& \left.-\frac{\varepsilon_{0}}{\mu} \dot{\bar{W}}_{1}(q, t)\right]=0,
\end{aligned}
$$

where

$$
\begin{aligned}
& h_{1}^{*}(t)=\sum_{r=1}^{\infty} \frac{\mu}{U_{c q}} \theta_{2}(q, r) \ddot{\bar{W}}_{1}(r, t), \\
& h_{2}^{*}(t)=\sum_{r=1}^{\infty} \frac{\mu}{U_{c r}} \theta_{2}(q, r) \ddot{\bar{W}}_{2}(r, t), \\
& h_{3}^{*}(t ; v) \\
& \quad=\ddot{\bar{W}}_{1}(r, t)\left[\theta_{1}(q, r)+2 \sum_{n=1}^{\infty} \cos \frac{n \pi v t}{L} \theta_{1 c}(q ; r)\right], \\
& h_{4}^{*}(t ; v) \\
& \quad=\ddot{\bar{W}}_{1}(r, t)\left[\theta_{3}(q, r)+2 \sum_{n=1}^{\infty} \cos \frac{n \pi v t}{L} \theta_{3 c}(q ; r)\right],
\end{aligned}
$$

$h_{5}^{*}(t ; v)$

$$
=\ddot{\bar{W}}_{1}(r, t)\left[\theta_{2}(q, r)+2 \sum_{n=1}^{\infty} \cos \frac{n \pi v t}{L} \theta_{2 c}(q ; r)\right],
$$

$\theta_{1}(q, r)=\int_{0}^{L} U_{r}(x) U_{q}(x) d x$

$\theta_{2}(q, r)=\int_{0}^{L} U_{r}(x) U_{q}^{\prime \prime}(x) d x$ 


$$
\begin{aligned}
& \theta_{3}(q, r)=\int_{0}^{L} U_{r}^{\prime}(x) U_{q}(x) d x, \\
& \theta_{1 c}(q, r)=\int_{0}^{L} \cos \frac{n \pi x}{L} U_{r}(x) U_{q}(x) d x, \\
& \theta_{2 c}(q, r)=\int_{0}^{L} \cos \frac{n \pi x}{L} U_{r}^{\prime \prime}(x) U_{q}(x) d x, \\
& \theta_{3 c}(q, r)=\int_{0}^{L} \cos \frac{n \pi x}{L} U_{r}^{\prime}(x) U_{q}(x) d x, \\
& U_{c r}=\int_{0}^{L} U_{r}^{2}(x) d x .
\end{aligned}
$$

Equations (16)-(25) are the coupled transformed secondorder ordinary differential equations governing the lateral behaviour of a double-Rayleigh beam system interconnected by a viscoelastic layer and traversed by a moving mass.

\subsection{Simplification of the Coupled Second-Order Differential} Equations. In general, it is difficult to get an exact analytical solution to a set of two coupled second-order ordinary differential equations unless certain simplifications are carried out and/or some assumptions are made. Furthermore, it is remarked at this juncture that the difficulty with (16), in particular, is not only that they are highly coupled but that the coefficients of the terms representing the inertia of the moving load are also functions of the independent variable $t$. Hence, it is expedient that this set of coupled, transformed second-order ordinary differential equations are simplified using certain assumptions. Specifically, the method of simplifying these equations involves following these three substeps: (a) Decouple the set of the ordinary differential equations. (b) Obtain modified frequency (I) due to the effect of rotatory inertia. (c) Obtain yet another modified frequency (II) due to the effect of the mass of the moving load.

\subsection{Partially Decoupled Transformed Ordinary Differential}

Equations. In this subsection, we seek to simplify the coupled transformed second-order ordinary differential equations (16). To this end, a system consisting of two unconnected Rayleigh beams is, in the time being, considered. It is also assumed that one of the beams (hereby referred to as first beam) is acted upon by a moving mass while the second vibrates freely. This system is a simplified version of system I and is, for convenience, hereby termed system II. For this type of system, (16) are reduced to

$$
\begin{aligned}
& \ddot{\bar{W}}_{1}(q, t)+\omega_{q}^{2} \bar{W}_{1}(q, t)-T_{\mathrm{ra1}} R_{I} h_{1}^{*}(t) \\
& +\frac{T_{\mathrm{ra} 3}}{L} \sum_{q=1}^{\infty} \frac{M_{L}}{U_{c q}}\left[h_{3}^{*}(t, v)+2 v h_{4}^{*}(t, v)+v^{2} h_{5}^{*}(t, v)\right] \\
& \quad=\frac{M_{L}}{\mu} g U_{q}(v t), \\
& \ddot{\bar{W}}_{2}(q, t)+\omega_{q}^{2} \bar{W}_{2}(q, t)-T_{\mathrm{ra1}} R_{I} h_{2}^{*}(t)=0 .
\end{aligned}
$$

Note that (26) and (27) can also be directly obtained from (16) by setting $T_{\text {ra2 }}$ to zero and are still coupled.

3.4. Method of Obtaining the Modified Frequency (I). Equations (26) and (27) are still difficult to solve. Hence, they have to be further simplified. To achieve this, the first Rayleigh beam of system II is hereby assumed, for the time being, to be acted upon by a moving force as opposed to a moving mass. In other words, the moving force problem of system II is considered. This amounts to setting $T_{\text {ra3 }}$ to zero in (26) so that (26) and (27) become

$$
\begin{aligned}
& \ddot{\bar{W}}_{1}(q, t)+\omega_{q}^{2} \bar{W}_{1}(q, t)-T_{\text {ral }} R_{I} h_{1}^{*}(t) \\
& \quad=\frac{M_{L}}{\mu} g U_{q}(v t), \\
& \ddot{W}_{2}(q, t)+\omega_{q}^{2} \bar{W}_{2}(q, t)-T_{\text {ral }} R_{I} h_{2}^{*}(t)=0,
\end{aligned}
$$

respectively.

Furthermore, it is still difficult to obtain an exact analytical solution to (28) and (29). Hence, one resorts to using an approximate analytical technique [1] which is a modification of the asymptotic method due to Struble $[1,3]$. This analytic technique involves obtaining a modified frequency (I) of the system due to the presence of the effect of rotatory inertia so that each of the differential operators in (28) and (29) is replaced by an equivalent operator defined by the modified frequency. Hence, following [1], one first denotes the ratio of the rotatory inertia correction factor, $R_{I}$, of any of the two beams to its length by $\gamma_{0}$ and define a small parameter $\alpha$ such that

$$
\alpha=\frac{\gamma_{0}}{1+\gamma_{0}}<1
$$

It follows, therefore, that

$$
\gamma_{0}=\alpha+0\left(\alpha^{2}\right) .
$$

Considering the homogenous part of (28), for instance, one obtains

$$
\begin{array}{r}
\ddot{\bar{W}}_{1}(q, t)+\omega_{q}^{2}\left(1+\alpha \theta^{*}(q, q)\right) \bar{W}_{1}(q, t) \\
-T_{\mathrm{ra} 1} \sum_{r=1, r \neq q}^{\infty} \alpha \theta^{*}(q, r) \ddot{\bar{W}}_{1}(r, t)=0,
\end{array}
$$

where

$$
\theta^{*}(q, q)=\frac{\mu L}{U_{c q}} \theta_{2}(q, q)
$$

According to Struble's technique [1,3], the general solution of (28) is of the form

$$
\begin{aligned}
\bar{W}_{1}(q, t)= & Z(q, t) \cos \left[\omega_{q} t-Q(q, t)\right] \\
& +\sum_{i=1}^{N_{1}} \alpha^{i} \bar{W}_{i}(q, t)+O\left(\alpha^{N_{1}+1}\right),
\end{aligned}
$$


where $Z(q, t)$ and $Q(q, t)$ are slowly time varying functions such that

$$
\begin{aligned}
& \dot{Z}(q, t) \approx 0(\alpha) ; \\
& \dot{Z}(q, t) \approx 0\left(\alpha^{2}\right), \\
& \dot{Q}(q, t) \approx 0(\alpha) ; \\
& \dot{Q}(q, t) \approx 0\left(\alpha^{2}\right)
\end{aligned}
$$

while $N_{1}$ is a finite natural number and " $\approx$ " denotes "is of order."

For $N_{1}=1$ and without loss of generality, we have

$$
\begin{aligned}
\bar{W}_{1}(q, t)= & Z(q, t) \cos \left[\omega_{q} t-Q(q, t)\right]+\alpha \bar{W}_{1}(q, t) \\
& +O\left(\alpha^{2}\right)
\end{aligned}
$$

and hence

$$
\begin{aligned}
& \ddot{\bar{W}}_{1}(q, t)=\left[\ddot{Z}(q, t)-\omega_{q}^{2} Z(q, t)\right. \\
& \left.+2 \omega_{q} Z(q, t) \dot{Q}(q, t)-Z(q, t) \dot{Q}^{2}(q, t)\right] \cos \left[\omega_{q} t\right. \\
& -Q(q, t)]-\left[2 \omega_{q} \dot{Z}(q, t)-2 \dot{Z}(q, t) \dot{Q}(q, t)\right. \\
& -Z(q, t) \ddot{Q}(q, t)] \sin \left[\omega_{q} t-Q(q, t)\right]+\alpha \ddot{\bar{W}}_{1}(q, t) \\
& +O\left(\alpha^{2}\right) .
\end{aligned}
$$

Substituting (36) and (37) into (32) and simplifying the resulting equation, taking into account (30), (31), and (35), we have

$$
\begin{aligned}
& 2 \omega_{q} Z(q, t) \dot{Q}(q, t) \cos \left[\omega_{q} t-Q(q, t)\right]-2 \omega_{q} \dot{Z}(q, t) \\
& \cdot \sin \left[\omega_{q} t-Q(q, t)\right]-2 \alpha v \omega_{q} Z(q, t) \theta_{2}(j, q) \\
& \cdot \sin \left[\omega_{q} t-Q(q, t)\right]-4 \alpha v \omega_{q} Z(q, t) \theta_{2}(j, q) \\
& \cdot \sum_{n=1}^{\infty} \cos \frac{n \pi v t}{L} \sin \left[\omega_{q} t-Q(q, t)\right]-\alpha \omega_{q}^{2} Z(q, t) \\
& \cdot \theta_{1}(j, q) \cos \left[\omega_{q} t-Q(q, t)\right]-2 \alpha \omega_{q}^{2} Z(q, t) \\
& \cdot \theta_{1}(j, q) \sum_{n=1}^{\infty} \cos \frac{n \pi v t}{L} \cos \left[\omega_{q} t-Q(q, t)\right] \\
& +\alpha v^{2} Z(q, t) \theta_{3}(j, q) \cos \left[\omega_{q} t-Q(q, t)\right] \\
& +2 \alpha v^{2} \theta_{3}(j, q) Z(q, t) \sum_{n=1}^{\infty} \cos \frac{n \pi v t}{L} \\
& \cdot \cos \left[\omega_{q} t-Q(q, t)\right]=0 .
\end{aligned}
$$

Neglecting terms which do not contribute to the variational equations, (38) becomes

$$
\begin{gathered}
\omega_{q}^{2} T_{\mathrm{ral}} \alpha \theta^{*}(q, q) Z(q, t) \cos \left[\omega_{q} t-Q(q, t)\right] \\
+2 \omega_{q} Z(q, t) \dot{Q}(q, t) \cos \left[\omega_{q} t-Q(q, t)\right] \\
-2 \omega \dot{Z}(q, t) \sin \left[\omega_{q} t-Q(q, t)\right]=0 .
\end{gathered}
$$

Setting the coefficients of $\cos \left[\omega_{q} t-Q(q, t)\right]$ and $\sin \left[\omega_{q} t-\right.$ $Q(q, t)]$ in (39) to zero, we have

$$
\begin{aligned}
2 \omega_{q} \dot{Q}(q, t)+\omega_{q}^{2} T_{\mathrm{ra} 1} \alpha \theta^{*}(q, q) & =0, \\
2 \omega_{q} \dot{Z}(q, t) & =0
\end{aligned}
$$

whose solutions are

$$
\begin{aligned}
& Z(q, t)=k_{0}, \\
& Q(q, t)=-\frac{\omega_{q}}{2}\left(\alpha T_{\mathrm{ral}} \theta^{*}(q, q) t\right)+k_{q},
\end{aligned}
$$

respectively, while $k_{0}, k_{q}$ are constants.

Hence, the desired modified frequency (I) due to the presence of rotatory inertia is

$$
\delta_{q}=\frac{\omega_{q}}{2}\left[2+T_{\mathrm{ra1}} \alpha \theta^{*}(q, q)\right]
$$

and the differential operator which acts on $W_{1}(q, t)$ and $W_{1}(r, t)$ in $(28)$ is, as earlier alluded to, replaced by the equivalent free system operator defined by the modified frequency, $\delta_{q}$. In other words, (28) which describes the transverse displacement of the first Rayleigh beam traversed by a moving force is reduced to

$$
\ddot{\bar{W}}_{1}(q, t)+\delta_{q}^{2} \bar{W}_{1}(q, t)=P_{\mathrm{OR}} U_{q}(v t),
$$

where

$$
P_{\mathrm{OR}}=\frac{M_{L} g}{\mu}\left(1+\alpha \theta^{*}(q, q)\right) .
$$

Using arguments similar to those presented thus far in this subsection, (29) is also reduced to

$$
\ddot{\bar{W}}_{2}(q, t)+\delta_{q}^{2} \bar{W}_{2}(q, t)=0 .
$$

The implication of (45) and (47) is that when the effect of the inertia terms (i.e., the terms involving the tracing constants $T_{\text {ra3 }}$ ) for system II is not neglected, (26) and (27) simplify to

$$
\begin{aligned}
& \ddot{\bar{W}}_{1}(q, t)+\delta_{q}^{2} \bar{W}_{1}(q, t)+T_{\mathrm{ra} 3} \frac{M_{L}}{\mu L} \sum_{q=1}^{\infty} \frac{1}{U_{c q}} \\
& \cdot\left[h_{2}^{*}(t, v)+2 v h_{3}^{*}(t, v)+v^{2} h_{4}^{*}(t, v)\right] \\
& =P_{\mathrm{OR}} U_{q}(v t), \\
& \ddot{\bar{W}}_{2}(q, t)+\delta_{q}^{2} \bar{W}_{2}(q, t)=0,
\end{aligned}
$$

respectively. In other words, the set of second-order ordinary differential equations for system II when the first Rayleigh beam is acted upon by a moving mass is now reduced to (48) and (49). 
3.5. Method of Obtaining the Modified Frequency (II). The problem of system II is now reduced to that of seeking the solutions to (48) and (49). It is however remarked at this juncture that while (49) can be easily solved, there is no exact analytical solution of (48). Hence, one again resorts to the approximate analytical method discussed in Section 3.4. The argument is that the problem under consideration involves the effect of both rotatory inertia and the inertia of the moving load. Hence, it is not sufficient to obtain the modified frequency (I) due to the effect of rotatory inertia only [2]. As a matter of fact, having obtained the modified frequency (I), $\delta_{q}$, we now proceed to obtain another frequency (a modified $\delta_{q}$ ), say $\Omega_{q}$, which is due not only to the presence of rotatory inertia but also to that of the moving mass. Following arguments similar to that of Section 3.4, an equivalent free system operator defined in terms of the new frequency, $\Omega_{q}$, for $\bar{W}_{1}(q, t)$ then replaces the terms on the left hand side of (48). To this end, we introduce a small parameter $\gamma$ such that

$$
\gamma=\frac{\gamma_{1}}{1+\gamma_{1}}<1
$$

where

$$
\gamma_{1}=\frac{M_{L}}{\mu L}
$$

is the mass ratio.

It also follows that

$$
\gamma_{1}=\gamma+0\left(\gamma^{2}\right)
$$

To obtain the desired modified frequency (II) due to the effect of the inertia of the moving load and that of the rotatory inertia, the homogeneous part of (48) is then considered. The first approximation to the assumed solution of the said homogeneous equation, according to Struble's technique, is

$$
\begin{aligned}
\bar{W}_{1}(q, t)= & Z(q, t) \cos \left[\delta_{q} t-Q(q, t)\right]+\gamma \bar{W}_{1}(q, t) \\
& +O\left(\gamma^{2}\right) .
\end{aligned}
$$

Again substituting (53) and its first- and second-order time derivatives into the homogeneous part of (48) taking into account (52), we have

$$
\begin{aligned}
- & 2 \delta_{q} \dot{Z}(q, t) \sin \left[\delta_{q} t-Q(q, t)\right]+2 Z(q, t) \dot{Q}(q, t) \\
\cdot & \delta_{q} \cos \left[\delta_{q} t-Q(q, t)\right]+\gamma \bar{W}_{1}(q, t) \\
+ & \gamma \delta_{q}^{2} \bar{W}_{1}(q, t)+\gamma \sum_{r=1}^{\infty} \frac{\mu}{U_{c r}} \\
\cdot & {\left[-\delta_{r}^{2}\left(\theta_{1}(r, t)+2 \sum_{n=1}^{\infty} \cos \frac{n \pi v t}{L}\right) \theta_{1 c}(q, r) Z(r, t)\right.} \\
\cdot & \cos \left[\delta_{r} t-Q(r, t)\right] \\
- & 2 \delta_{r} v\left(\theta_{3}(q, r)+2 \sum_{n=1}^{\infty} \cos \frac{n \pi v t}{L} \theta_{3 c}(q, r)\right) Z(r, t)
\end{aligned}
$$

$$
\begin{aligned}
& \cdot \sin \left[\delta_{r} t-Q(r, t)\right] \\
& +v^{2}\left(\theta_{2}(q, r)+2 \sum_{n=1}^{\infty} \cos \frac{n \pi v t}{L} \theta_{2 c}(q, r)\right) Z(r, t) \\
& \left.\cdot \cos \left[\delta_{r} t-Q(r, t)\right]\right]=0
\end{aligned}
$$

where the terms in $\gamma^{2}$ and higher power of $\gamma$ have been neglected. The corresponding variational equations are

$$
\begin{array}{r}
-2 \delta_{q} \dot{Z}(q, t)-2 \frac{\gamma v \mu \delta_{q}}{U_{c r}} \theta_{3}(q, q) Z(q, t)=0, \\
2 Z \dot{Q}(q, t) \delta_{q}-\frac{\gamma \delta_{q}^{2}}{U_{c r}} \mu\left(\theta_{1}(q, q)-v^{2} \frac{\theta_{2}(q, q)}{\delta_{q}^{2}}\right)=0 .
\end{array}
$$

Solving (55), we have

$$
\begin{aligned}
& Z(q, t)=A^{0} e^{-r^{0}} t \\
& Q(q, t)=\frac{\mu \gamma \delta_{q}}{2 U_{c r}}\left(\theta_{1}(q, q)-v^{2} \frac{\theta_{2}(q, q)}{\delta_{q}^{2}}\right) t+\phi_{q}
\end{aligned}
$$

where $r^{0}=\mu \gamma v \theta_{3}(q, q) / U_{c r}, A^{0}$ and $\phi_{q}$ are constants, and hence

$$
\Omega_{q}=\delta_{q}\left[1-\frac{\mu \gamma}{2 U_{c r}}\left(\theta_{1}(q, q)-\frac{v^{2} \theta_{2}(q, q)}{\delta_{q}^{2}}\right)\right] .
$$

Equation (57) is the desired modified frequency (II) corresponding to the frequency of the free system involving rotatory inertia and moving mass effect.

Hence, according to Struble's technique, (48) reduces to

$$
\ddot{\bar{W}}_{1}(q, t)+\Omega_{q}^{2} \bar{W}_{1}(q, t)=P_{T R} U_{q}(v t)
$$

where

$$
P_{T R}=\gamma g L
$$

and the set of second-order transformed ordinary differential equations for system II are now made up of (45) and (58) whose closed form solutions can be obtained without much difficulty.

3.6. Solution of the Two Viscoelastically Connected Rayleigh Beams. Recall that system II is a simplified version of the original system I and it is obtained by assuming that the two Rayleigh beams are not joined by a layer (i.e., $T_{\mathrm{ra} 2}=0$ ). Now, if the viscoelastic layer is retained, then $T_{\mathrm{ra} 2}$ is not equal to 
zero. In this case and in view of (45) and (58), the two secondorder transformed coupled ordinary differential equations for the double-Rayleigh beam system I are finally simplified to

$$
\begin{aligned}
& \ddot{\bar{W}}_{1}(q, t)+\Omega_{q}^{2} \bar{W}_{1}(q, t)+T_{\mathrm{ra} 2}\left[\frac{k_{1}}{\mu} \bar{W}_{1}(q, t)\right. \\
& \left.+\frac{\varepsilon_{0}}{\mu} \dot{\bar{W}}_{1}(q, t)-\frac{k_{1}}{\mu} \bar{W}_{2}(q, t)-\frac{\varepsilon_{0}}{\mu} \dot{\bar{W}}_{2}(q, t)\right] \\
& \quad=P_{\mathrm{OR}} U_{q}(v t), \\
& \ddot{\bar{W}_{2}}(q, t)+\delta_{q}^{2} \bar{W}_{2}(q, t)+T_{\mathrm{ra} 2}\left[\frac{k_{1}}{\mu} \bar{W}_{1}(q, t)\right. \\
& \left.+\frac{\varepsilon_{0}}{\mu} \dot{\bar{W}}_{1}(q, t)-\frac{k_{1}}{\mu} \bar{W}_{2}(q, t)-\frac{\varepsilon_{0}}{\mu} \dot{W}_{2}(q, t)\right]=0
\end{aligned}
$$

in terms of the two modified frequencies $\left(\delta_{q}\right.$ and $\left.\Omega_{q}\right)$, respectively. In other words, problem of assessing the dynamic behaviour of a double-Rayleigh beam system I traversed by a moving mass under arbitrary end supports reduces to that of solving (60) subjected to the corresponding transformed initial conditions. To solve the coupled differential equations (60) a semianalytical method known as differential transform method (DTM) is used. To this end, we first state briefly the basic theory of the method as follows. The differential transform of the $m$ th derivative of a function $w(t)$ is given as $[28,29]$

$$
W(m)=\frac{1}{m !}\left[\frac{d^{m} w(t)}{d t^{m}}\right]_{t=t_{0}}
$$

The corresponding inverse transformation is defined as

$$
w(t)=\sum_{m=0}^{\infty}\left(t-t_{0}\right)^{m} W(m) .
$$

Hence, (61) and (62) yield

$$
w(t)=\sum_{m=0}^{\infty} \frac{\left(t-t_{0}\right)^{m}}{m !}\left[\frac{d^{m} w(t)}{d t^{m}}\right]_{t=t_{0}}
$$

It is well known that, in application, the series in (62) is finite and usually written as

$$
w(t)=\sum_{m=0}^{P}\left(t-t_{0}\right)^{m} W(m)
$$

\begin{tabular}{|c|c|}
\hline Original function & T-function \\
\hline$w(t)=u(t) \pm v(t)$ & $\bar{W}(k)=\bar{U}(k) \pm \bar{V}(k)$ \\
\hline$w(t)=c u(t)$ & $\bar{W}(k)=c \bar{U}(k)$ \\
\hline$w(t)=\frac{d u(t)}{d t}$ & $\bar{W}(k)=(k+1) \bar{U}(k+1)$ \\
\hline$w(t)=\frac{d^{n} u(t)}{d t^{n}}$ & $\begin{array}{c}\bar{W}(k)= \\
(k+1)(k+2) \cdots(k+n- \\
1)(k+n) \bar{U}(k+n) \\
k\end{array}$ \\
\hline$w(t)=u(t) v(t)$ & $\begin{array}{c}\bar{W}(k)=\sum_{n=0}^{k} \bar{U}(n) \bar{V}(k-n) \\
\bar{W}(k)=\end{array}$ \\
\hline$w(t)=u(t) v(t) y(t)$ & $\sum_{n=0}^{k} \sum_{r=0}^{k-n} \bar{U}(n) \bar{V}(r) \bar{Y}(k-n-r)$ \\
\hline$w(t)=$ & $\begin{array}{ll}\bar{W}(k)= \\
k & \bar{V}(n)\end{array}$ \\
\hline$u(t) \int_{0}^{L} v(x) d t$ & $\sum_{n=1}^{\kappa} \bar{U}(k-n) \frac{V(n-1)}{n} k \geq 1$ \\
\hline$w(t)=t^{m}$ & $\begin{array}{c}\bar{W}(k)=\delta(k-m)= \\
\begin{cases}1, & k=m \\
0, & k \neq m\end{cases} \end{array}$ \\
\hline$w(t)=\sin a t$ & $\bar{W}(k)=\frac{1}{k !} a^{k} \sin \left(\frac{k \pi}{2}\right)$ \\
\hline$w(t)=\cos a t$ & $\bar{W}(k)=\frac{1}{k !} a^{k} \cos \left(\frac{k \pi}{2}\right)$ \\
\hline$w(t)=\sinh a t$ & $\bar{W}(k)=\frac{1}{2 k !}\left[(a)^{k}-(-a)^{k}\right]$ \\
\hline$w(t)=\cosh a t$ & $\bar{W}(k)=\frac{1}{2 k !}\left[(a)^{k}+(-a)^{k}\right]$ \\
\hline
\end{tabular}

such that the series $\sum_{m=P+1}^{\infty}\left(t-t_{0}\right)^{m} W(m)$ is considered unimportantly small.

Furthermore, it can be readily shown $[28,29]$ that the relationships in Table 1 between the original function $w(t)$ and the transformed function $W(m)$, for $t_{0}=0$, hold.
TABLE 1: Basic theorems of DTM for equations of motion.

Next, the application of the differential transform on (60), using Table 1, yields the following recurrence relations for $m \geq 0$

$\bar{W}_{1}(m+2)$

$$
\begin{aligned}
& =\frac{1}{(m+1)(m+2)}\left[P _ { \mathrm { OR } } \left[\frac{1}{m !}\left(\frac{\lambda_{q} v}{L}\right)^{m} \sin \left(\frac{m \pi}{2}\right)\right.\right. \\
& +\frac{A_{q}}{m !}\left(\frac{\lambda_{q} v}{L}\right)^{m} \cos \left(\frac{m \pi}{2}\right) \\
& +\frac{B_{q}}{2 m !}\left[\left(\frac{\lambda_{q} v}{L}\right)^{m}-\left(-\frac{\lambda_{q} v}{L}\right)^{m}\right] \\
& \left.+\frac{C_{q}}{2 m !}\left[\left(\frac{\lambda_{q} v}{L}\right)^{m}+\left(-\frac{\lambda_{q} v}{L}\right)^{m}\right]\right]-\Omega_{q}^{2} \bar{W}_{1}(m) \\
& -T_{\mathrm{ra} 2} \frac{k_{1}}{\mu} \bar{W}_{1}(m)-T_{\mathrm{ra} 2} \frac{\varepsilon_{0}}{\mu}(m+1) \bar{W}_{1}(m+1) \\
& \left.+T_{\mathrm{ra} 2} \frac{k_{1}}{\mu} \bar{W}_{2}(m)+T_{\mathrm{ra} 2} \frac{\varepsilon_{0}}{\mu}(m+1) \bar{W}_{2}(m+1)\right],
\end{aligned}
$$


Chinese Journal of Mathematics

9

$$
\begin{gathered}
\bar{W}_{2}(m+2)=\frac{1}{(m+1)(m+2)}\left[-\delta_{q}^{2} \bar{W}_{2}(m)-T_{\mathrm{ra} 2}\right. \\
. \frac{k_{1}}{\mu} \bar{W}_{2}(m)-T_{\mathrm{ra} 2} \frac{\varepsilon_{0}}{\mu}(m+1) \bar{W}_{2}(m+1)+T_{\mathrm{ra} 2} \\
\left.. \frac{k_{1}}{\mu} \bar{W}_{1}(m)+T_{\mathrm{ra} 2} \frac{\varepsilon_{0}}{\mu}(m+1) \bar{W}_{1}(m+1)\right] .
\end{gathered}
$$

Note that, in obtaining (65), (12) had been used. Equations (60) are also to be solved subject to the following transformed initial conditions:

$$
\begin{aligned}
& \bar{W}_{1}(0)=0=\bar{W}_{1}(1), \\
& \bar{W}_{2}(0)=0=\bar{W}_{2}(1) .
\end{aligned}
$$

Substituting (67) for $m=0,1,2,3, \ldots$ into recurrence relations (65), (66), using "MAPLE 18," we have

$$
\begin{aligned}
& \bar{W}_{1}(2)=\frac{P_{\mathrm{OR}}}{2 !}\left(A_{q}+C_{q}\right), \\
& \bar{W}_{2}(2)=0 \text {, } \\
& \bar{W}_{1}(3)=\frac{P_{\mathrm{OR}}}{3 !}\left[\sigma_{q}\left(1+B_{q}\right)-T_{\mathrm{ra} 2} \frac{\varepsilon_{0}}{\mu}\left(A_{q}+C_{q}\right)\right] \text {, } \\
& \sigma_{q}=\frac{\lambda_{q} v}{L} \\
& \bar{W}_{2}(3)=\frac{P_{\mathrm{OR}}}{3 !} T_{\mathrm{ra} 2} \frac{\varepsilon_{0}}{\mu}\left(A_{q}+C_{q}\right) \\
& \bar{W}_{1}(4)=\frac{P_{\mathrm{OR}}}{4 !}\left[\left(\sigma_{q}^{2}-\Omega_{q}^{2}\right) C_{q}-\left(\sigma_{q}^{2}+\Omega_{q}^{2}\right) A_{q}\right. \\
& -T_{\mathrm{ra} 2} \frac{k_{1}}{\mu}\left(A_{q}+C_{q}\right)-T_{\mathrm{ra} 2} \frac{\varepsilon_{0}}{\mu} \sigma_{q}\left(1+B_{q}\right) \\
& \left.+2 \frac{T_{\mathrm{ra} 2}^{2} \varepsilon_{0}^{2}}{\mu^{2}}\left(A_{q}+C_{q}\right)\right] \\
& \bar{W}_{2}(4)=\frac{P_{\mathrm{OR}}}{4 !}\left[-2 T_{\mathrm{ra} 2}^{2} \frac{\varepsilon_{0}^{2}}{\mu^{2}}\left(A_{q}+C_{q}\right)\right. \\
& \left.+T_{\mathrm{ra} 2} \frac{k_{1}}{\mu}\left(A_{q}+C_{q}\right)+T_{\mathrm{ra} 2} \frac{\varepsilon_{0}}{\mu} \sigma_{q}\left(1+B_{q}\right)\right] \text {, } \\
& \bar{W}_{1}(5)=\frac{P_{\mathrm{OR}}}{5 !}\left[\sigma_{q}^{3}\left(B_{q}-1\right)+\Omega_{q}^{2} T_{\mathrm{ra} 2} \frac{\varepsilon_{0}}{\mu}\left(A_{q}+C_{q}\right)\right. \\
& -\Omega_{q}^{2} \sigma_{q}\left(1+B_{q}\right)-\frac{T_{\mathrm{ra} 2} k_{1}}{\mu} \sigma_{q}\left(1+B_{q}\right) \\
& +4 \frac{T_{\mathrm{ra} 2}^{2} k_{1} \varepsilon_{0}}{\mu^{2}}\left(A_{q}+C_{q}\right)-\frac{T_{\mathrm{ra} 2} \varepsilon_{0}}{\mu} C_{q}\left(\sigma_{q}^{2}-\Omega_{q}^{2}\right) \\
& +\frac{T_{\mathrm{ra} 2} \varepsilon_{0}}{\mu} A_{q}\left(\sigma_{q}^{2}+\Omega_{q}^{2}\right)+2 \frac{T_{\mathrm{ra} 2}^{2} \varepsilon_{0}^{2}}{\mu^{2}} \sigma_{q}\left(1+B_{q}\right) \\
& \left.-4 \frac{T_{\mathrm{ra} 2}^{3} \varepsilon_{0}^{3}}{\mu^{3}}\left(A_{q}+C_{q}\right)\right]
\end{aligned}
$$

Using the inverse differential transform of (64) in conjunctimon with (67), for $t_{0}=0$, we obtain

$$
\begin{aligned}
& W_{1 m m}(x, t)=\sum_{q=1}^{\infty} \frac{P_{R T} \mu}{U_{c q}\left(\sigma_{q}^{2}-\Omega_{q}^{2}\right)} \\
& \left\{\left[\frac{1}{2 !}\left(A_{q}+C_{q}\right)\left(\sigma_{q}^{2}-\Omega_{q}^{2}\right)\right] t^{2}\right. \\
& +\frac{\left(\sigma_{q}^{2}-\Omega_{q}^{2}\right)^{2}}{3 !}\left[\sigma_{q}\left(1+B_{q}\right)-\frac{T_{\mathrm{ra} 2} \varepsilon_{0}}{\mu}\left(A_{q}+C_{q}\right)\right] \\
& \cdot t^{3}+\frac{\left(\sigma_{q}^{2}-\Omega_{q}^{2}\right)^{2}}{4 !}\left[C_{q}-\frac{\left(\sigma_{q}^{2}+\Omega_{q}^{2}\right)}{\left(\sigma_{q}^{2}-\Omega_{q}^{2}\right)} A_{q}\right. \\
& -\frac{T_{\mathrm{ra} 2} k_{1}}{\mu} \frac{\left(A_{q}+C_{q}\right)}{\left(\sigma_{q}^{2}-\Omega_{q}^{2}\right)}-\frac{T_{\mathrm{ra} 2} \varepsilon_{0} \sigma_{q}}{\mu} \frac{\left(1+B_{q}\right)}{\left(\sigma_{q}^{2}-\Omega_{q}^{2}\right)} \\
& \left.+\frac{2 T_{\mathrm{ra} 2}^{2} \varepsilon_{0}^{2}}{\mu^{2}} \frac{\left(A_{q}+C_{q}\right)}{\left(\sigma_{q}^{2}-\Omega_{q}^{2}\right)}\right] t^{4} \\
& +\frac{\left(\sigma_{q}^{2}-\Omega_{q}^{2}\right)^{2}}{5 !}\left[-\frac{\sigma_{q}^{3}\left(B_{q}-1\right)}{\left(\sigma_{q}^{2}-\Omega_{q}^{2}\right)}\right. \\
& +\frac{\Omega_{q}^{2} T_{\mathrm{ra} 2} \varepsilon_{0}\left(A_{q}+C_{q}\right)}{\mu\left(\sigma_{q}^{2}-\Omega_{q}^{2}\right)}-\frac{\Omega_{q}^{2} \sigma_{q}\left(1+B_{q}\right)}{\left(\sigma_{q}^{2}-\Omega_{q}^{2}\right)} \\
& -\frac{T_{\mathrm{ra} 2} k_{1} \sigma_{q}\left(1+B_{q}\right)}{\mu\left(\sigma_{q}^{2}-\Omega_{q}^{2}\right)}+\frac{4 T_{\mathrm{ra} 2}^{2} k_{1} \varepsilon_{0}\left(A_{q}+C_{q}\right)}{\mu^{2}\left(\sigma_{q}^{2}-\Omega_{q}^{2}\right)} \\
& -\frac{T_{\mathrm{ra} 2} \varepsilon_{0} C_{q}}{\mu}+\frac{T_{\mathrm{ra} 2} \varepsilon_{0} A_{q}\left(\sigma_{q}^{2}+\Omega_{q}^{2}\right)}{\mu\left(\sigma_{q}^{2}-\Omega_{q}^{2}\right)} \\
& \left.+\frac{2 T_{\mathrm{ra} 2}^{2} \varepsilon_{0}^{2} \sigma_{q}\left(1+B_{q}\right)}{\mu^{2}\left(\sigma_{q}^{2}-\Omega_{q}^{2}\right)}-\frac{4 T_{\mathrm{ra} 2}^{3} \varepsilon_{0}^{3}\left(A_{q}+C_{q}\right)}{\mu^{3}\left(\sigma_{q}^{2}-\Omega_{q}^{2}\right)}\right] t^{5} \\
& +\cdots\}\left[\sin \frac{\lambda_{q}}{L} x+A_{q} \cos \frac{\lambda_{q}}{L} x+B_{q} \sinh \frac{\lambda_{q}}{L} x\right. \\
& \left.+C_{q} \cosh \frac{\lambda_{q}}{L} x\right] \text {, }
\end{aligned}
$$




$$
\begin{aligned}
& W_{2 m m}(x, t)=\sum_{q=1}^{\infty} \frac{P_{R T} \mu}{U_{c q}\left(\sigma_{q}^{2}-\delta_{q}^{2}\right)} \\
& \left\{\frac{1}{3 !}\left[\frac{T_{\mathrm{ra} 2} \varepsilon_{0}}{\mu}\left(A_{q}+C_{q}\right)\left(\sigma_{q}^{2}-\delta_{q}^{2}\right)\right] t^{3}\right. \\
& +\frac{\left(\sigma_{q}^{2}-\delta_{q}^{2}\right)}{4 !}\left[\frac{T_{\mathrm{ra} 2} k_{1}}{\mu}\left(A_{q}+C_{q}\right)\right. \\
& \left.+\frac{T_{\mathrm{ra} 2} \varepsilon_{0} \sigma_{q}}{\mu}\left(1+B_{q}\right)-2 \frac{T_{\mathrm{ra} 2}^{2} \varepsilon_{0}^{2}}{\mu^{2}}\left(A_{q}+C_{q}\right)\right] t^{4} \\
& +\frac{\left(\sigma_{q}^{2}-\delta_{q}^{2}\right)^{2}}{5 !}\left[\frac{T_{\mathrm{ra} 2} \varepsilon_{0} C_{q}}{\mu}\left(\sigma_{q}^{2}-\delta_{q}^{2}\right)\right. \\
& -\frac{T_{\mathrm{ra} 2} \varepsilon_{0} A_{q}}{\mu}\left(\sigma_{q}^{2}+\delta_{q}^{2}\right)+\frac{T_{\mathrm{ra} 2} k_{1} \sigma_{q}}{\mu}\left(1+B_{q}\right) \\
& -\frac{T_{\mathrm{ra} 2} \varepsilon_{0} \Omega_{q}^{2}}{\mu}\left(A_{q}+C_{q}\right)-4 \frac{T_{\mathrm{ra} 2}^{2} k_{1} \varepsilon_{0}}{\mu^{2}}\left(A_{q}+C_{q}\right) \\
& \left.-2 \frac{T_{\mathrm{ra} 2}^{2} \varepsilon_{0}^{2} \sigma_{q}}{\mu^{2}}\left(1+B_{q}\right)+4 \frac{T_{\mathrm{ra} 2}^{3} \varepsilon_{0}^{3}}{\mu^{3}}\left(A_{q}+C_{q}\right)\right] t^{5} \\
& +\cdots\}\left[\sin \frac{\lambda_{q}}{L} x+A_{q} \cos \frac{\lambda_{q}}{L} x+B_{q} \sinh \frac{\lambda_{q}}{L} x\right. \\
& \left.+C_{q} \cosh \frac{\lambda_{q}}{L} x\right] \text {. }
\end{aligned}
$$

Equations (76) represent the transverse displacements of the double-Rayleigh beams interconnected by a viscoelastic layer, traversed by a moving mass and having arbitrary end supports.

For the purpose of comparison the moving force problem associated with system I is considered. It is therefore remarked at this juncture that in view of (45), (46), and (47) and retaining the viscoelastic core, the two reduced transformed coupled second-order ordinary differential equations for the corresponding moving force problem of system I having arbitrary boundary conditions are

$$
\begin{aligned}
& \ddot{\bar{W}}_{1}(q, t)+\delta_{q}^{2} \bar{W}_{1}(q, t)+T_{\text {ra } 2}\left[\frac{k_{1}}{\mu} \bar{W}_{1}(q, t)\right. \\
& \left.+\frac{\varepsilon_{0}}{\mu} \dot{W}_{1}(q, t)-\frac{k_{1}}{\mu} \bar{W}_{2}(q, t)-\frac{\varepsilon_{0}}{\mu} \dot{\bar{W}}_{2}(q, t)\right] \\
& \quad=P_{R T} U_{q}(v t),
\end{aligned}
$$

$$
\begin{aligned}
& \ddot{\bar{W}}_{2}(q, t)+\delta_{q}^{2} \bar{W}_{2}(q, t)+T_{\mathrm{ra} 2}\left[\frac{k_{1}}{\mu} \bar{W}_{2}(q, t)\right. \\
& \left.+\frac{\varepsilon_{0}}{\mu} \dot{\bar{W}}_{2}(q, t)-\frac{k_{1}}{\mu} \bar{W}_{1}(q, t)-\frac{\varepsilon_{0}}{\mu} \dot{\bar{W}}_{1}(q, t)\right]=0 .
\end{aligned}
$$

Solving (77), subject to the corresponding transformed initial conditions, using the differential transform method, one obtains, after inversion, the following:

$$
\begin{aligned}
& W_{1 m f}(x, t)=\sum_{q=1}^{\infty} \frac{\mu P_{R T}}{U_{c q}\left(\sigma_{q}^{2}-\delta_{q}^{2}\right)}\left[\frac { 1 } { 2 ! } ( A _ { q } + C _ { q } ) \left(\sigma_{q}^{2}\right.\right. \\
& \left.-\delta_{q}^{2}\right) t^{2}+\frac{\left(\sigma_{q}^{2}-\delta_{q}^{2}\right)}{3 !}\left[\sigma_{q}\left(1+B_{q}\right)\right. \\
& \left.-\frac{T_{\mathrm{ra} 2} \varepsilon_{0}}{\mu}\left(A_{q}+C_{q}\right)\right] t^{3}+\frac{\left(\sigma_{q}^{2}-\delta_{q}^{2}\right)^{2}}{4 !}\left[C_{q}\right. \\
& -\frac{\left(\sigma_{q}^{2}+\delta_{q}^{2}\right)}{\left(\sigma_{q}^{2}-\delta_{q}^{2}\right)} A_{q}-\frac{T_{\mathrm{ra} 2} k_{1}}{\mu} \frac{\left(A_{q}+C_{q}\right)}{\left(\sigma_{q}^{2}-\delta_{q}^{2}\right)} \\
& \left.-\frac{T_{\mathrm{ra} 2} \varepsilon_{0} \sigma_{q}}{\mu} \frac{\left(1+B_{q}\right)}{\left(\sigma_{q}^{2}-\delta_{q}^{2}\right)}+2 \frac{T_{\mathrm{ra} 2}^{2} \varepsilon_{0}^{2}}{\mu^{2}} \frac{\left(A_{q}+C_{q}\right)}{\left(\sigma_{q}^{2}-\delta_{q}^{2}\right)}\right] t^{4} \\
& +\frac{\left(\sigma_{q}^{2}-\delta_{q}^{2}\right)^{2}}{5 !}\left[\frac{\sigma_{q}^{3}\left(B_{q}-1\right)}{\left(\sigma_{q}^{2}-\delta_{q}^{2}\right)}+\delta_{q}^{2} \frac{T_{\mathrm{ra} 2} \varepsilon_{0}}{\mu} \frac{\left(A_{q}+C_{q}\right)}{\left(\sigma_{q}^{2}-\delta_{q}^{2}\right)}\right. \\
& -\delta_{q}^{2} \frac{\sigma_{q}\left(1+B_{q}\right)}{\left(\sigma_{q}^{2}-\delta_{q}^{2}\right)}-\frac{T_{\mathrm{ra} 2} k_{1} \sigma_{q}\left(1+B_{q}\right)}{\mu\left(\sigma_{q}^{2}-\delta_{q}^{2}\right)} \\
& \left.\left.+4 \frac{T_{\mathrm{ra} 2}^{2} k_{1} \varepsilon_{0}\left(A_{q}+C_{q}\right)}{\mu^{2}\left(\sigma_{q}^{2}-\delta_{q}^{2}\right)}\right]+\cdots\right]\left[\sin \frac{\lambda_{q}}{L} x+A_{q}\right. \\
& \left.\cdot \cos \frac{\lambda_{q}}{L} x+B_{q} \sinh \frac{\lambda_{q}}{L} x+C_{q} \cosh \frac{\lambda_{q}}{L} x\right] \text {, } \\
& W_{2 m f}(x, t)=\sum_{q=1}^{\infty} \frac{\mu P_{R T}}{U_{c q}\left(\sigma_{q}^{2}-\delta_{q}^{2}\right)} \\
& \cdot\left[\frac{1}{3 !}\left[\frac{T_{\mathrm{ra} 2} \varepsilon_{0}}{\mu}\left(A_{q}+C_{q}\right)\left(\sigma_{q}^{2}-\delta_{q}^{2}\right)\right] t^{3}\right. \\
& +\frac{\left(\sigma_{q}^{2}-\delta_{q}^{2}\right)}{4 !}\left[\frac{T_{\mathrm{ra} 2} k_{1}}{\mu}\left(A_{q}+C_{q}\right)\right. \\
& \left.+\frac{T_{\mathrm{ra} 2} \varepsilon_{0} \sigma_{q}}{\mu}\left(1+B_{q}\right)-2 \frac{T_{\mathrm{ra} 2}^{2} \varepsilon_{0}^{2}}{\mu^{2}}\left(A_{q}+C_{q}\right)\right] t^{4} \\
& +\frac{\left(\sigma_{q}^{2}-\delta_{q}^{2}\right)}{5 !}\left[\frac{T_{\mathrm{ra} 2} \varepsilon_{0} C_{q}}{\mu}\left(\sigma_{q}^{2}-\delta_{q}^{2}\right)\right. \\
& -\frac{T_{\mathrm{ra} 2} \varepsilon_{0}}{\mu} A_{q}\left(\sigma_{q}^{2}+\delta_{q}^{2}\right)+\frac{T_{\mathrm{ra} 2} k_{1}}{\mu} C_{q}\left(1+B_{q}\right) \\
& -\frac{T_{\mathrm{ra} 2} \varepsilon_{0} \delta_{q}^{2}}{\mu}\left(A_{q}+C_{q}\right)-4 \frac{T_{\mathrm{ra} 2}^{2} k_{1} \varepsilon_{0}}{\mu^{2}}\left(A_{q}+C_{q}\right)
\end{aligned}
$$




$$
\begin{aligned}
& \left.-2 \frac{T_{\mathrm{ra} 2}^{2} \varepsilon_{0}^{2}}{\mu^{2}} \sigma_{q}\left(1+B_{q}\right)+4 \frac{T_{\mathrm{ra} 2}^{3} \varepsilon_{0}^{3}}{\mu^{3}}\left(A_{q}+C_{q}\right)\right] t^{5} \\
& +\cdots]\left[\sin \frac{\lambda_{q}}{L} x+A_{q} \cos \frac{\lambda_{q}}{L} x+B_{q} \sinh \frac{\lambda_{q}}{L} x\right. \\
& \left.+C_{q} \cosh \frac{\lambda_{q}}{L} x\right] .
\end{aligned}
$$

Equations (78) and (79) denote the lateral deflections of the upper and lower Rayleigh beams, respectively, due to concentrated moving force having constant velocity and traversing a viscoelastically connected double-Rayleigh beam system with general end supports.

\section{Some Case Studies}

Hitherto, the discussion has been for general boundary conditions. In this section, two case studies involving (i) simply supported end conditions and (ii) clamped-clamped end conditions are considered in order to illustrate the theory developed thus far.

4.1. Simply Supported Double-Rayleigh Beam System. The system considered here comprises two finite Rayleigh beams which are simply supported and are interconnected by a viscoelastic layer. The boundary conditions for such simply supported double-Rayleigh beam system are [3, 22, 24]

$$
\begin{gathered}
W_{1}(0 ; t)=W_{2}(0 ; t)=0 ; \\
W_{1}(L ; t)=W_{2}(L ; t)=0 \\
W_{1}^{\prime \prime}(0 ; t)=W_{2}^{\prime \prime}(0 ; t)=0 ; \\
W_{1}^{\prime \prime}(L ; t)=W_{2}^{\prime \prime}(L ; t)=0
\end{gathered}
$$

Hence, for the eigenfunction, $U_{q}(x)$, we have

$$
\begin{aligned}
& U_{q}(0)=0=U_{q}(L), \\
& U_{q}^{\prime \prime}(0)=0=U_{q}^{\prime \prime}(L)
\end{aligned}
$$

as well as

$$
\begin{gathered}
U_{r}(0)=0=U_{r}(L), \\
U_{r}^{\prime \prime}(0)=0=U_{r}^{\prime \prime}(L) .
\end{gathered}
$$

It follows, therefore, that in view of (12), (80), and (81) we have

$$
\begin{aligned}
A_{q} & =B_{q}=C_{q}=0=A_{r}=B_{r}=C_{r}, \\
\sin \lambda_{q} & =0=\sin \lambda_{r},
\end{aligned}
$$

so that

$$
\begin{aligned}
& \lambda_{q}=q \pi ; \\
& \lambda_{r}=r \pi ;
\end{aligned}
$$

Equation (15) implies

$$
\begin{aligned}
& \omega_{q}^{2}=\frac{q^{4} \pi^{4}}{L^{4}} \frac{E I}{\mu}, \\
& \omega_{r}^{2}=\frac{r^{4} \pi^{4}}{L^{4}} \frac{E I}{\mu}
\end{aligned}
$$

and the corresponding eigenfunction reduces to

$$
U_{q}(x)=\sin \frac{\lambda_{q} x}{L} .
$$

The associated initial conditions are as given in (7). Hence the generalized finite transforms, (8) and (9) and their inverses, (10) and (11) as well as (13) reduce to

$$
\begin{aligned}
\bar{W}_{1}(q, t) & =\int_{0}^{L} W_{1}(x, t) \sin \frac{\lambda_{q} x}{L} d x, \\
\bar{W}_{2}(q, t) & =\int_{0}^{L} W_{2}(x, t) \sin \frac{\lambda_{q} x}{L} d x, \\
W_{1}(x, t) & =\sum_{q=1}^{\infty} \frac{\mu}{U_{c q s}} \bar{W}_{1}(q, t) \sin \frac{\lambda_{q} x}{L}, \\
W_{2}(x, t) & =\sum_{q=1}^{\infty} \frac{\mu}{U_{c q s}} \bar{W}_{2}(q, t) \sin \frac{\lambda_{q} x}{L}, \\
U_{c q s} & =\int_{0}^{L} \frac{\sin ^{2}}{\lambda_{q} x} d x,
\end{aligned}
$$

respectively.

Substituting (86) into (22)-(25) and the resulting expressions into (17)-(21) while the results of the latter are in turn substituted into the transformed equations (16), one obtains

$$
\begin{aligned}
& \left(1+T_{\mathrm{ra} 1} R_{I} \frac{q^{2} \pi^{2}}{L^{2}}\right) \ddot{\bar{W}}_{1}(q, t)+\omega_{q}^{2} \bar{W}_{1}(q, t) \\
& +T_{\mathrm{ra} 3} \varepsilon_{2}\left[\Gamma_{0}(q ; v)\right. \\
& \left.+\sum_{q=0}^{\infty}\left[\Gamma_{1}(q, v)-16 \sum_{j=i}^{\infty} \Gamma_{2}(q, v)-\Gamma_{3}(q, v)\right]\right] \\
& +T_{r a 2}\left[\frac{k_{1}}{\mu} \bar{W}_{1}(q, t)+\frac{\varepsilon_{0}}{\mu} \dot{\bar{W}}_{1}(q, t)-\frac{k_{1}}{\mu} \bar{W}_{2}(q, t)\right. \\
& \left.-\frac{\varepsilon_{0}}{\mu} \dot{\bar{W}}_{2}(q, t)\right]=\frac{M_{L} g}{\mu} \sin \frac{q \pi v t}{L}, \\
& \left(1+T_{\mathrm{ra} 1} R_{I} \frac{q^{2} \pi^{2}}{L^{2}}\right) \ddot{\bar{W}}_{2}(q, t)+\omega_{q}^{2} \bar{W}_{2}(q, t) \\
& +T_{\mathrm{ra} 2}\left[\frac{k_{1}}{\mu} \bar{W}_{2}(q, t)+\frac{\varepsilon_{0}}{\mu} \dot{\bar{W}}_{2}(q, t)-\frac{k_{1}}{\mu} \bar{W}_{1}(q, t)\right. \\
& \left.-\frac{\varepsilon_{0}}{\mu} \dot{\bar{W}}_{1}(q, t)\right]=0
\end{aligned}
$$


where

$$
\begin{aligned}
& \Gamma_{0}(q, v)=\ddot{\bar{W}}_{1}(q, t)-\left(\frac{v^{2} q^{2} \pi^{2}}{L^{2}}\right) \bar{W}_{1}(q, t), \\
& \Gamma_{1}(q, v)=2 \sin \left(\frac{q \pi v t}{L}\right) \sin \left(\frac{r \pi v t}{L}\right) \ddot{\bar{W}}_{1}(q, t) \\
& \quad-\frac{8 v r q \pi^{2}}{r^{2} \pi^{2}-q^{2} v^{2}} \dot{\bar{W}}_{1}(r, t), \\
& \Gamma_{2}(q, v)=\frac{v r q \pi^{4}\left(j^{2}+r^{2}-q^{2}\right) \cos j \pi v t}{\left((j+r)^{2} \pi^{2}-q^{2} \pi^{2}\right)\left((j-r)^{2} \pi^{2}-q^{2} \pi^{2}\right)} \\
& \quad \cdot \dot{\bar{W}}_{1}(r, t), \\
& \Gamma_{3}(q, v)=\left(\frac{2 v^{2} r^{2} \pi^{2}}{L^{2}}\right) \sin \left(\frac{q \pi v t}{L}\right) \sin \left(\frac{r \pi v t}{L}\right) \\
& \cdot W_{1}(r, t), \\
& \varepsilon_{2}=\frac{M_{L}}{\mu L}=\gamma_{1} .
\end{aligned}
$$

Equations (92) and (93) constitute the coupled transformed second-order ordinary differential equations describing the transverse response of a simply supported doubleRayleigh beam system interconnected by a viscoelastic layer and traversed by a moving mass. Note that (92) can also be obtained by applying (87)-(91) on (1)-(3) using the initial conditions (7). Following the discussion in Section 3.3 and in particular for simply supported system II, (92) and (101) reduce to

$$
\begin{aligned}
& \left(1+T_{\mathrm{ra} 1} R_{I} \frac{q^{2} \pi^{2}}{L^{2}}\right) \ddot{\bar{W}}_{1}(q, t)+\omega_{q}^{2} \bar{W}_{1}(q, t) \\
& +T_{\mathrm{ra} 3} \varepsilon_{2}\left[\Gamma_{0}(q ; v)\right. \\
& \left.+\sum_{q=0}^{\infty}\left[\Gamma_{1}(q, v)-16 \sum_{j=i}^{\infty} \Gamma_{2}(q, v)-\Gamma_{3}(q, v)\right]\right] \\
& =\frac{M_{L} g}{\mu} \sin \frac{q \pi v t}{L}, \\
& \left(1+T_{\mathrm{ra} 1} R_{I} \frac{q^{2} \pi^{2}}{L^{2}}\right) \ddot{\bar{W}}_{2}(q, t)+\omega_{q}^{2} \bar{W}_{2}(q, t)=0,
\end{aligned}
$$

respectively.

Also, by following arguments similar to those of Section 3.4, it is firstly assumed that the simply supported system II is traversed by a moving force. Thus, (94) and (95) reduce to

$$
\begin{aligned}
& \ddot{W}_{1}(q, t)+\omega_{q s s}^{2} \bar{W}_{1}(q, t)=L^{*} \sin \left(\frac{q \pi v t}{L}\right), \\
& \ddot{W}_{2}(q, t)+\omega_{q s s}^{2} \bar{W}_{2}(q, t)=0,
\end{aligned}
$$

respectively, where

$$
\begin{gathered}
\omega_{q s s}^{2}=\frac{\omega_{q}^{2}}{\left(1+T_{\mathrm{ra} 1} R_{I}\left(q^{2} \pi^{2} / L^{2}\right)\right)} ; \\
L^{*}=\frac{\left(M_{L} g / \mu\right)}{\left(1+T_{\mathrm{ra} 1} R_{I}\left(q^{2} \pi^{2} / L^{2}\right)\right)} .
\end{gathered}
$$

Note that solutions to (96) and (97) can easily be obtained and are well known [1]. As a matter of fact, there is no need, for the simply supported system II traversed by a moving force, to obtain the modified frequency (I) due to the effect of rotatory inertia.

Next, we consider the case in which the simply supported system II is traversed by a moving mass as opposed to a moving force. In other words, we seek to simplify the entire (94) and (95) when $T_{\text {ra3 }} \neq 0$. To this end, we again use modified Struble's method and following arguments similar to those in Section 3.5, we obtain

$$
\begin{aligned}
\gamma_{q s s} & =\frac{\omega_{q s s}}{2}\left[1+\frac{1}{1+T_{\mathrm{ral}} R_{I}\left(\left(q^{2} \pi^{2}\right) / L^{2}\right)}\right. \\
- & \left.\frac{2 \gamma\left(\omega_{q s s}^{2}+\left(v^{2} q^{2} \pi^{2}\right) / L^{2}\right)}{\omega_{q s s}^{2}\left(1+T_{\mathrm{ral}} R_{I}\left(\left(q^{2} \pi^{2}\right) / L^{2}\right)\right)}\right]
\end{aligned}
$$

which is the modified frequency II corresponding to the presence of the moving mass and hence (94) and (95) reduce to

$$
\begin{aligned}
& \ddot{\bar{W}}_{1}(q, t)+\gamma_{q s s}^{2} \bar{W}_{1}(q, t) \\
& \quad=\frac{\gamma g L}{1+T_{\mathrm{ral}} R_{I}\left(q^{2} \pi^{2} / L^{2}\right)} \sin \left(\frac{q \pi v t}{L}\right), \\
& \ddot{\bar{W}}_{2}(q, t)+\omega_{q s s}^{2} \bar{W}_{2}(q, t)=0 .
\end{aligned}
$$

Hence, the two second-order transformed coupled ordinary differential equations for a simply supported viscoelastically interconnected double-Rayleigh beam system I are

$$
\begin{gathered}
\ddot{\bar{W}}_{1}(q, t)+\gamma_{q s s}^{2} \bar{W}_{1}(q, t)+T_{\mathrm{ra} 2}\left[\frac{k_{1}}{\mu} \bar{W}_{1}(q, t)\right. \\
\left.+\frac{\varepsilon_{0}}{\mu} \dot{\bar{W}}_{1}(q, t)-\frac{k_{1}}{\mu} \bar{W}_{2}(q, t)-\frac{\varepsilon_{0}}{\mu} \dot{\bar{W}}_{2}(q, t)\right] \\
=\frac{\gamma g L}{1+T_{\mathrm{ra} 1} R_{I}\left(q^{2} \pi^{2} / L^{2}\right)} \sin \left(\frac{q \pi v t}{L}\right), \\
\ddot{\bar{W}}_{2}(q, t)+\omega_{q s s}^{2} \bar{W}_{2}(q, t)+T_{\mathrm{ra} 2}\left[\frac{k_{1}}{\mu} \bar{W}_{2}(q, t)\right. \\
\left.+\frac{\varepsilon_{0}}{\mu} \dot{\bar{W}}_{2}(q, t)-\frac{k_{1}}{\mu} \bar{W}_{1}(q, t)-\frac{\varepsilon_{0}}{\mu} \dot{\bar{W}}_{1}(q, t)\right]=0 .
\end{gathered}
$$


Solving (101) using differential transform method, we obtain

$$
\begin{aligned}
& W_{1 s s m}(x, t)=\sum_{q=1}^{\infty}\left(\frac{\eta g l}{1+T_{\mathrm{ral}} R_{I}\left(q^{2} \pi^{2} / l^{2}\right)}\right) \\
& \cdot \frac{\mu \sigma_{q} s s}{\left(\sigma_{q s s}^{2}-\gamma_{q s s m}^{2}\right) U_{q s s}}\left[\frac{\left(\sigma_{q s s}^{2}-\gamma_{q s s m}^{2}\right)}{3 !} t^{3}\right. \\
& -\frac{\left(\sigma_{q s s}^{2}-\gamma_{q s s m}^{2}\right) T_{\mathrm{ra} 2} \varepsilon_{0}}{4 ! \mu} t^{4}-\frac{\left(\sigma_{q s s}^{2}-\gamma_{q s s m}^{2}\right)}{5 !}\left(\gamma_{q s s m}^{2}\right. \\
& \left.+\frac{T_{\mathrm{ra} 2} k_{1}}{\mu}-\frac{2 T_{\mathrm{ra} 2} \varepsilon_{0}^{2}}{\mu^{2}}+\sigma_{q s s}^{2}\right) t^{5} \\
& +\frac{\left(\sigma_{q s s}^{2}-\gamma_{q s s m}^{2}\right)}{6 !}\left(\frac{2 \gamma_{q s s m}^{2} T_{\mathrm{ra} 2} \varepsilon_{0}}{\mu}+\frac{4 T_{\mathrm{ra} 2}^{2} k_{1} \varepsilon_{0}}{\mu^{2}}\right. \\
& \left.\left.-\frac{4 T_{\mathrm{ra} 2}^{3} \varepsilon_{0}^{3}}{\mu^{3}}-\sigma_{q s s}^{2}\right) t^{6}+\cdots\right] \sin \frac{q \pi x}{L}, \\
& W_{2 s s m}(x, t)=\sum_{q=1}^{\infty}\left(\frac{\sigma_{q s s} \mu g l \eta}{1+T_{\mathrm{ral}} R_{I}\left(q^{2} \pi^{2} / l^{2}\right)}\right) \\
& \cdot \frac{1}{\left(\sigma_{q s s}^{2}-\omega_{q s s m}^{2}\right) U_{q s s}}\left[\frac{\left(\sigma_{q s s}^{2}-\omega_{q s s m}^{2}\right)}{4 !} \frac{T_{\mathrm{ra} 2} \varepsilon_{0}}{\mu} t^{4}\right. \\
& +\frac{\left(\sigma_{q s s}^{2}-\omega_{q s s m}^{2}\right)}{5 !}\left(\frac{T_{\mathrm{ra} 2} k_{1}}{\mu}-\frac{2 T_{\mathrm{ra} 2}^{2} \varepsilon_{0}^{2}}{\mu^{2}}\right) t^{5} \\
& +\frac{\left(\sigma_{q s s}^{2}-\omega_{q s s m}^{2}\right)}{6 !}\left[-\left(\frac{\omega_{q s s m}^{2} T_{\mathrm{ra} 2} \varepsilon_{0}}{\mu}-\frac{3 T_{\mathrm{ra} 2}^{2} k_{1} \varepsilon_{0}}{\mu^{2}}\right.\right. \\
& +\frac{2 T_{\mathrm{ra} 2}^{3} \varepsilon_{0}^{3}}{\mu^{3}}-\frac{\gamma_{q s s m}^{2} T_{\mathrm{ra} 2} \varepsilon_{0}}{\mu}-\frac{T_{\mathrm{ra} 2} k_{1}}{\mu}+2 \frac{T_{\mathrm{ra} 2}^{2} \varepsilon_{0}^{2}}{\mu^{2}} \\
& \left.\left.\left.-\sigma_{q}^{2}\right)\right] t^{6}+\cdots\right] \sin \frac{q \pi x}{L} .
\end{aligned}
$$

Equations (102) and (103) represent the lateral deflections of the simply supported double-Rayleigh beam traversed by a moving mass for both the upper, $W_{1 s s m}(x, t)$, and the lower, $W_{2 s s m}(x, t)$, viscoelastically interconnected Rayleigh beams, respectively.

Furthermore, setting $T_{\text {ra3 }}$ to zero in (92), one obtains the following reduced coupled transformed second-order ordinary differential equations describing the vibration of simply supported viscoelastically connected double-Rayleigh beams under a concentrated moving force:

$$
\begin{aligned}
\ddot{\bar{W}}_{1 s s f}(q, t)+\omega_{q s s}^{2} \bar{W}_{1 s s f}(q, t)+N^{*}\left[\frac{k_{1}}{\mu} \bar{W}_{1 s s f}(q, t)\right. \\
+\frac{\varepsilon_{0}}{\mu} \dot{\bar{W}}_{1 s s f}(q, t)-\frac{k_{1}}{\mu} \bar{W}_{2 s s f}(q, t) \\
\left.-\frac{\varepsilon_{0}}{\mu} \dot{\bar{W}}_{2 s s f}(q, t)\right]=L^{*} \sin \frac{q \pi v t}{L},
\end{aligned}
$$

$$
\begin{aligned}
& \ddot{\bar{W}}_{2 s s f}(q, t)+\omega_{q s s}^{2} \bar{W}_{2 s s f}(q, t)+N^{*}\left[\frac{k_{1}}{\mu} \bar{W}_{2 s s f}(q, t)\right. \\
& \quad+\frac{\varepsilon_{0}}{\mu} \dot{\bar{W}}_{2 s s f}(q, t)-\frac{k_{1}}{\mu} \bar{W}_{1 s s f}(q, t) \\
& \left.\quad-\frac{\varepsilon_{0}}{\mu} \dot{\bar{W}}_{1 s s f}(q, t)\right]=0
\end{aligned}
$$

where

$$
N^{*}=\frac{T_{\mathrm{ra} 2}}{1+T_{\mathrm{ra} 1} R_{I}\left(q^{2} \pi^{2} / L^{2}\right)} .
$$

The dynamic response of the simply supported viscoelastically interconnected double-Rayleigh beam system to a moving force after a number of simplifications is found to be

$$
\begin{aligned}
& W_{1 s s f}(x, t)=\sum_{q=1}^{\infty} \frac{L^{*} \sigma_{q s s}\left(\sigma_{q s s}^{2}-\omega_{q s s f}^{2}\right)}{3}\left[\frac{\left(\sigma_{q}^{2}-\omega_{q s s f}^{2}\right)}{3 !} t^{3}\right. \\
& -\frac{\varepsilon_{0} N^{*}\left(\sigma_{q s s}^{2}-\omega_{q s s f}^{2}\right)}{4 ! \mu} t^{4}-\frac{\left(\sigma_{q s s}^{2}-\omega_{q s s f}^{2}\right)}{5 !}\left[\omega_{q s s f}^{2}\right. \\
& \left.+\frac{N^{*} k_{1}}{\mu}-\frac{2 N^{* 2} \varepsilon_{0}^{2}}{\mu^{2}}+\sigma_{q s s}^{2}\right] t^{5} \\
& . \frac{\left(\sigma_{q s s}^{2}-\omega_{q s s f}^{2}\right)}{6 !}\left[\frac{2 \omega_{q s s f}^{2} N^{*} \varepsilon_{0}}{\mu}+\frac{4 N^{* 2} k_{1} \varepsilon_{0}}{\mu^{2}}\right. \\
& \left.\left.-\frac{4 N^{* 3} \varepsilon_{0}^{3}}{\mu^{3}}-\frac{N^{*} \varepsilon_{0}}{\mu} \sigma_{q}^{2}\right] t^{6}+\cdots\right] \sin \frac{q \pi x}{L}
\end{aligned}
$$

for the upper Rayleigh beam while the lateral deflection for the lower Rayleigh beam is represented by

$$
\begin{aligned}
& W_{2 s s f}(x, t)=\sum_{q=1}^{\infty} \frac{L^{*} N^{*} \sigma_{q s s}\left(\sigma_{q s s}^{2}-\omega_{q s s f}^{2}\right)}{U_{0 s}} \\
& {\left[\frac{\varepsilon_{0}\left(\sigma_{q s s}^{2}-\omega_{q s s f}^{2}\right)}{\mu 4 !} t^{4}+\frac{\left(\sigma_{q s s}^{2}-\omega_{q s s f}^{2}\right)}{5 !}\left[\frac{k_{1}}{\mu}\right.\right.} \\
& \left.-\frac{2 N^{*} \varepsilon_{0}^{2}}{\mu^{2}}\right] t^{5}+\frac{\left(\sigma_{q s s}^{2}-\omega_{q s s f}^{2}\right)}{6 !}\left[-2 \omega_{q s s f}^{2} \frac{\varepsilon_{0}}{\mu}\right. \\
& \left.\left.-\frac{4 N^{*} k_{1} \varepsilon_{0}}{\mu^{2}}+\frac{4 N^{* 2} \varepsilon_{0}^{3}}{\mu^{3}}-\frac{\varepsilon_{0} \sigma_{q s s}^{2}}{\mu}\right] t^{6}+\cdots\right] \sin \frac{q \pi x}{L} .
\end{aligned}
$$

4.2. Clamped-Clamped Double-Rayleigh Beam System. Next we consider the second case study involving a clampedclamped double-Rayleigh beam system connected by a viscoelastic core and traversed by a moving load. For such a system having this type of vibrating configuration, both its 
deflection and slope vanish at the ends and the corresponding boundary conditions are

$$
\begin{aligned}
& W_{1}(0 ; t)=W_{2}(0 ; t)=0 ; \\
& W_{1}(L ; t)=W_{2}(L ; t)=0, \\
& W_{1}^{\prime}(0 ; t)=W_{2}^{\prime}(0 ; t)=0 ; \\
& W_{1}^{\prime}(L ; t)=W_{2}^{\prime}(L ; t)=0 .
\end{aligned}
$$

In terms of the eigenfunctions $U_{q}(x)$, these boundary conditions become

$$
\begin{gathered}
U_{q}(0)=0=U_{q}(L), \\
U_{q}^{\prime}(0)=0=U_{q}^{\prime}(L) .
\end{gathered}
$$

Similarly, it follows that

$$
\begin{gathered}
U_{r}(0)=0=U_{r}(L), \\
U_{r}^{\prime}(0)=0=U_{r}^{\prime}(L) .
\end{gathered}
$$

Now applying (109) on (12), it follows that

$$
\begin{aligned}
& A_{q}=-C_{q}=-\frac{\sinh \lambda_{q}-\sin \lambda_{q}}{\cosh \lambda_{q}-\cos \lambda_{q}}=A_{r}=-C_{r}, \\
& B_{q}=-1=B_{r}
\end{aligned}
$$

and the particular eigenfunction for this vibrating configuration is

$$
\begin{aligned}
U_{q m c}(x)= & \cosh \frac{\lambda_{q} x}{L}-\cos \frac{\lambda_{q} x}{L} \\
& -\sigma_{q}\left(\sinh \frac{\lambda_{q} x}{L}-\sin \frac{\lambda_{q} x}{L}\right),
\end{aligned}
$$

where

$$
\sigma_{q}=\frac{\cosh \lambda_{q}-\cos \lambda_{q}}{\sinh \lambda_{q}-\sin \lambda_{q}}=\frac{1}{C_{q}} .
$$

The corresponding frequency equation is

$$
\cosh \lambda_{q}=\frac{1}{\cos \lambda_{q}}
$$

whose roots are [2]

$$
\begin{aligned}
& \lambda_{1}=4.73004 ; \\
& \lambda_{2}=7.85320 ; \\
& \lambda_{3}=10.99561 ;
\end{aligned}
$$

The corresponding initial conditions remain the same as those prescribed in (7).

Two limiting cases of the forced vibrating problem are considered in what follows. These are
(1) the moving mass problem involving the clampedclamped double finite Rayleigh beam system which are viscoelastically connected with the effect of inertia of the moving load being taken into consideration,

(2) the moving force problem consisting of two finite Rayleigh beams viscoelastically interconnected, clamped at both ends, and traversed by a moving load whose inertia is negligible.

In view of (60) and (112), the pair of governing transformed, coupled, second-order ordinary differential equations for case 1 above is

$$
\begin{aligned}
& \ddot{\bar{W}}_{1 m c}(q, t)+\Omega_{q m c}^{2} \bar{W}_{1 m c}(q, t)+T_{\mathrm{ra} 2}\left[\frac{k_{1}}{\mu} \bar{W}_{1 m c}(q, t)\right. \\
& +\frac{\varepsilon_{0}}{\mu} \dot{\bar{W}}_{1 m c}(q, t)-\frac{k_{1}}{\mu} \bar{W}_{2 m c}(q, t) \\
& \left.\quad-\frac{\varepsilon_{0}}{\mu} \dot{\bar{W}}_{2 m c}(q, t)\right]=P_{R T} U_{q m c}(v t), \\
& \ddot{\bar{W}}_{2 m c}(q, t)+\delta_{q m c}^{2} \bar{W}_{2 m c}(q, t)+T_{\mathrm{ra} 2}\left[\frac{k_{1}}{\mu} \bar{W}_{2 m c}(q, t)\right. \\
& +\frac{\varepsilon_{0}}{\mu} \dot{\bar{W}}_{2 m c}(q, t)-\frac{k_{1}}{\mu} \bar{W}_{1 m c}(q, t) \\
& \left.-\frac{\varepsilon_{0}}{\mu} \dot{\bar{W}}_{1 m c}(q, t)\right]=0
\end{aligned}
$$

where

$$
\begin{aligned}
& \Omega_{q m c} \\
& =\delta_{q m c}\left[1-\frac{\mu \gamma}{2 U_{q m c}}\left(\theta_{1 m c}(q, q)-\frac{v^{2} \theta_{2 m c}(q, q)}{\delta_{q m c}^{2}}\right)\right] \\
& \delta_{q m c}=\frac{\omega_{q m c}}{2}\left[2+T_{\mathrm{ra} 1} \alpha \theta^{*}(q, q)\right] \\
& \theta^{*}(q, q)=\frac{\mu L}{U_{q m c}} \theta_{2 m c}(q, q) ; \\
& \alpha=\frac{R_{I}}{L} ; \\
& \gamma=\frac{M}{\mu L}, \\
& \theta_{q m c}(q, q)=\int_{0}^{L} U_{q m c}(x) U_{q m x}(x) d x \\
& \theta_{2 m c}(q, q)=\int_{0}^{L} U_{q m c}^{\prime \prime}(x) U_{q m c}(x) d x
\end{aligned}
$$


while the corresponding equations for the second case are

$$
\begin{aligned}
& \ddot{\bar{W}}_{1 f c}(q, t)+\delta_{q f c}^{2} \bar{W}_{1 f c}(q, t)+T_{\mathrm{ra} 2}\left[\frac{k_{1}}{\mu} \bar{W}_{1 f c}(q, t)\right. \\
& \left.+\frac{\varepsilon_{0}}{\mu} \dot{\bar{W}}_{1 f c}(q, t)-\frac{k_{1}}{\mu} \bar{W}_{2 f c}(q, t)-\frac{\varepsilon_{0}}{\mu} \dot{\bar{W}}_{2 f c}(q, t)\right] \\
& =P_{R T} U_{q m c}(v t), \\
& \ddot{\bar{W}}_{2 f c}(q, t)+\delta_{q f c}^{2} \bar{W}_{2 f c}(q, t)+T_{\mathrm{ra} 2}\left[\frac{k_{1}}{\mu} \bar{W}_{2 f c}(q, t)\right. \\
& \left.+\frac{\varepsilon_{0}}{\mu} \dot{\bar{W}}_{2 f c}(q, t)-\frac{k_{1}}{\mu} \bar{W}_{1 f c}(q, t)-\frac{\varepsilon_{0}}{\mu} \dot{\bar{W}}_{1 f c}(q, t)\right] \\
& =0,
\end{aligned}
$$

where

$$
\delta_{q f c}=\delta_{q m c} .
$$

Solving the differential equations (116) subject to the corresponding transformed initial conditions (67) using DTM and following arguments similar to those in Section 3.6 for the moving force and moving mass gives, respectively, after inversion

$$
\begin{aligned}
& W_{1 m c}(x, t)=\sum_{q=1}^{\infty} \frac{P_{R T} \sigma_{q m c}^{2}}{\mu U_{q m c}\left(\delta_{q m c}^{2}-\Omega_{q m c}^{2}\right)}\left[\frac{2 \mu\left(\delta_{q m c}^{2}-\Omega_{q m c}^{2}\right)}{4 !}\right. \\
& \cdot t^{2}-2\left(\delta_{q m c}^{2}-\Omega_{q m c}^{2}\right)\left(\frac{\mu \delta_{q m c} \sigma_{q}+\varepsilon_{0} T_{\mathrm{ra} 2}}{5 !}\right) t^{3}-2\left(\delta_{q m c}^{2}\right. \\
& \left.-\Omega_{q m c}^{2}\right) \\
& \cdot\left(\frac{\mu \Omega_{q m c}^{2}+T_{\mathrm{ra} 2}\left(k_{1}-\varepsilon_{0} \delta_{q m c} \sigma_{q m c}-\varepsilon_{0} / \mu\right)+\varepsilon_{0}^{2} T_{\mathrm{ra} 2} / \mu}{6 !}\right) \\
& \cdot t^{4}+\frac{\left(\delta_{q m c}^{2}-\Omega_{q m c}^{2}\right)}{7 !}\left[-2 \Omega_{q m c}^{2}\left(-\mu \delta_{q m c} \sigma_{q m c}+\varepsilon_{0} T_{\mathrm{ra} 2}\right)\right. \\
& -2 T_{\mathrm{ra} 2}\left(k_{1} \delta_{q m c} \sigma_{q m c}-\varepsilon_{0} T_{\mathrm{ra} 2}\right)+\varepsilon_{0}\left(-2 \Omega_{q m c}^{2}\right. \\
& -2 T_{\mathrm{ra} 2}\left(k_{1}-\varepsilon_{0} \delta_{q m c} \sigma_{q m c}-\frac{\varepsilon_{0}}{\mu}-\frac{2 \varepsilon_{0}^{2} T_{\mathrm{ra} 2}^{2}}{\mu}\right)+\frac{2 k_{1} \varepsilon_{0} T_{\mathrm{ra} 2}}{\mu} \\
& \left.\left.+\frac{\varepsilon_{0}^{3} T_{\mathrm{ra} 2}^{2}}{\mu^{2}}-\frac{2 k_{1} \varepsilon_{0} T_{\mathrm{ra} 2}}{\mu}+\varepsilon_{0}^{2} \delta_{q m c} T_{\mathrm{ra} 2} \sigma_{q m c}+T_{\mathrm{ra} 2} \varepsilon_{0}^{2} \sigma_{q}\right)\right] t^{5} \\
& +\cdots] U_{q m c}(x),
\end{aligned}
$$

$$
\begin{aligned}
& W_{2 m c}(x, t)=\sum_{q=1}^{\infty} \frac{P_{R T} \sigma_{q m c}^{2}}{\mu U_{q m c}\left(\sigma_{q m c}^{2}-\delta_{q m c}^{2}\right)}\left[\frac{1}{5 !}\left(2 \varepsilon_{0} T_{\mathrm{ra} 2}\right) t^{3}\right. \\
& -\frac{\left(\sigma_{q m c}^{2}-\delta_{q m c}^{2}\right)}{6 !}\left(\frac{2 \varepsilon_{0}^{2} T_{\mathrm{ra} 2}^{2}}{\mu}-2 k_{1} T_{\mathrm{ra} 2}+\varepsilon_{0} \delta_{q m c} T_{\mathrm{ra} 2} \sigma_{q m c}\right. \\
& \left.-\frac{\varepsilon_{0} T_{\mathrm{ra} 2}^{2}}{\mu}+\varepsilon_{0} T_{\mathrm{ra} 2} \sigma_{q m c}\right) t^{4} \frac{\left(\sigma_{q m c}^{2}-\delta_{q m c}^{2}\right)}{7 !}\left[2 \varepsilon_{0} \delta_{q m c}^{2}\right. \\
& +T_{\mathrm{ra} 2}\left[\frac{2 k_{1} \varepsilon_{0}}{\mu}-2 \frac{\varepsilon_{0}^{3} T_{\mathrm{ra} 2}^{2}}{\mu^{2}}+2 \frac{k_{1} \varepsilon_{0} T_{\mathrm{ra} 2}}{\mu}-\frac{\varepsilon_{0}^{2} \delta_{q m c} T_{\mathrm{ra} 2} \sigma_{q m c}}{\mu}\right. \\
& \left.-\frac{\varepsilon_{0}^{2} \sigma_{q m c}}{\mu}-\frac{\varepsilon_{0}^{3} T_{\mathrm{ra} 2}^{2}}{\mu^{2}}\right]-2 k_{1} \delta_{q m c} \sigma_{q m c}-2 \frac{k_{1} \varepsilon_{0} T_{\mathrm{ra} 2}}{\mu} \\
& -2 \Omega_{q m c}^{2} \varepsilon_{0}-\varepsilon_{0} T_{\mathrm{ra} 2}\left[2 \frac{k_{1}}{\mu}-2 \frac{\varepsilon_{0} \delta_{q m c} \sigma_{q m c}}{\mu}-2 \frac{\varepsilon_{0}}{\mu^{2}}\right. \\
& \left.\left.\left.-2 \frac{\varepsilon_{0}^{2} T_{\mathrm{ra} 2}}{\mu^{2}}\right]\right] t^{5}+\cdots\right] U_{q m c}(x) .
\end{aligned}
$$

Equations (120) are the lateral displacements for the clampedclamped double-Rayleigh beam system traversed by a moving mass. Solving (118) together with the initial conditions using DTM, one obtains

$$
\begin{aligned}
& W_{1 f c}(x, t)=\sum_{q=1}^{\infty} \frac{P_{\mathrm{OR}} \sigma_{q}^{2}}{\mu U_{q f c}\left(\sigma_{q f c}^{2}-\delta_{q f c}^{2}\right)}\left[\frac{2 \mu\left(\delta_{q f c}^{2}-\Omega_{q f c}^{2}\right)}{4 !}\right. \\
& \cdot t^{2}-2\left(\sigma_{q f c}^{2}-\delta_{q f c}^{2}\right)\left(\frac{\mu \delta_{q f c} \sigma_{q f c}+\varepsilon_{0} T_{\mathrm{ra} 2}}{5 !}\right) t^{3} \\
& -2\left(\sigma_{q f c}^{2}-\delta_{q f c}^{2}\right) \\
& \cdot\left(\frac{\mu \delta_{q f c}^{2}+T_{\mathrm{ra} 2}\left(k_{1}-\varepsilon_{0} \delta_{q f c} \sigma_{q f c}-\varepsilon_{0} / \mu\right)+\varepsilon_{0}^{2} T_{\mathrm{ra} 2} / \mu}{6 !}\right) \\
& \cdot t^{4}+\frac{\left(\sigma_{q f c}^{2}-\delta_{q f c}^{2}\right)}{7 !}\left[-2 \delta_{q f c}^{2}\left(-\mu \delta_{q f c} \sigma_{q f c}+\varepsilon_{0} T_{\mathrm{ra} 2}\right)\right. \\
& -2 T_{\mathrm{ra} 2}\left(k_{1} \delta_{q f c} \sigma_{q f c}-\varepsilon_{0} T_{\mathrm{ra} 2}\right)+\varepsilon_{0}\left(-2 \delta_{q f c}^{2}\right. \\
& -2 T_{\mathrm{ra} 2}\left(k_{1}-\varepsilon_{0} \delta_{q f c} \sigma_{q f c}-\frac{\varepsilon_{0}}{\mu}-\frac{2 \varepsilon_{0}^{2} T_{\mathrm{ra} 2}^{2}}{\mu}\right) \\
& +\frac{2 k_{1} \varepsilon_{0} T_{\mathrm{ra} 2}}{\mu}+\frac{\varepsilon_{0}^{3} T_{\mathrm{ra} 2}^{2}}{\mu^{2}}-\frac{2 k_{1} \varepsilon_{0} T_{\mathrm{ra} 2}}{\mu}+\varepsilon_{0}^{2} \delta_{q f c} T_{\mathrm{ra} 2} \sigma_{q f c} \\
& \left.\left.\left.+T_{\mathrm{ra} 2} \varepsilon_{0}^{2} \sigma_{q f c}\right)\right] t^{5}+\cdots\right] U_{q f c}(x),
\end{aligned}
$$




$$
\begin{aligned}
W_{2 f c}(x, t)=\sum_{q=1}^{\infty} \frac{P_{\mathrm{OR}} \sigma_{q f c}^{2}}{\mu U_{q f c}\left(\sigma_{q f c}^{2}-\omega_{q f c}^{2}\right)}\left[\frac{1}{5 !}\left(2 \varepsilon_{0} T_{\mathrm{ra} 2}\right) t^{3}\right. \\
-\frac{\left(\sigma_{q f c}^{2}-\omega_{q f c}^{2}\right)}{6 !}\left(\frac{2 \varepsilon_{0}^{2} T_{\mathrm{ra} 2}^{2}}{\mu}-2 k_{1} T_{\mathrm{ra} 2}+\varepsilon_{0} \delta_{q f c} T_{\mathrm{ra} 2} \sigma_{q f c}\right. \\
-\frac{\left.\varepsilon_{0} T_{\mathrm{ra} 2}^{2}+\varepsilon_{0} T_{\mathrm{ra} 2} \sigma_{q f c}\right) t^{4} \frac{\left(\sigma_{q f c}^{2}-\omega_{q f c}^{2}\right)}{\mu !}\left[2 \varepsilon_{0} \omega_{q f c}^{2}\right.}{\mu} \\
+T_{\mathrm{ra} 2}\left[\frac{2 k_{1} \varepsilon_{0}}{\mu}-2 \frac{\varepsilon_{0}^{3} T_{\mathrm{ra} 2}^{2}}{\mu^{2}}+2 \frac{k_{1} \varepsilon_{0} T_{\mathrm{ra} 2}}{\mu}\right. \\
\left.-\frac{\varepsilon_{0}^{2} \delta_{q f c} T_{\mathrm{ra} 2} \sigma_{q f c}}{\mu}-\frac{\varepsilon_{0}^{2} \sigma_{q f c}}{\mu}-\frac{\varepsilon_{0}^{3} T_{\mathrm{ra} 2}^{2}}{\mu^{2}}\right]-2 k_{1} \delta_{q f c} \sigma_{q f c} \\
\left.-2 \frac{k_{1} \varepsilon_{0} T_{\mathrm{ra} 2}-2 \delta_{q f c}^{2} \varepsilon_{0}-\varepsilon_{0} T_{\mathrm{ra} 2}\left[2 \frac{k_{1}}{\mu}-2 \frac{\varepsilon_{0} \delta_{q f c} \sigma_{q f c}}{\mu}\right.}{\mu}\right] t_{q f c}(x) . \\
\left.\left.-2 \frac{\varepsilon_{0}}{\mu^{2}}-2 \frac{\varepsilon_{0}^{2} T_{\mathrm{ra} 2}}{\mu^{2}}\right]\right]
\end{aligned}
$$

Equations (121) represent the lateral displacements for the clamped-clamped double-Rayleigh beam system traversed by a moving force.

\section{Resonance Conditions}

It should be recalled that, for the system under consideration, damping effect has been assumed negligible. The dynamic deflections of the double-Rayleigh beam system may, therefore, grow beyond bounds. In other words, the system may be in a state of resonance. Hence, it becomes necessary to investigate the conditions under which the system reaches a state of resonance and the speed of the moving load at which this phenomenon of resonance takes place. Such a speed is usually referred to as critical speed while the corresponding conditions are known as resonance conditions.

Specifically, for the dynamic response of a simply supported viscoelastically interconnected double-Rayleigh beam system due to moving mass, (102) and (103) show that the phenomenon of resonance will be developed in the system when

$$
\begin{gathered}
\gamma_{q s s m}=\sigma_{q s s}, \text { for the upper beam, } \\
\sigma_{q s s}=\omega_{q s s m}, \text { for the lower beam }
\end{gathered}
$$

while when the same system is traversed by a moving force (106) and (107) yield the corresponding resonance condition

$$
\sigma_{q s s}=\omega_{q s s f}
$$

It is noted also that $\sigma_{q s s}=v \pi q / L$, so that in view of (122) and (123), for example, $V_{c r}=\left(\omega_{q s s} L\right) / \pi q$, where $V_{c r}$ is the critical velocity of the simply supported double-Rayleigh beam due to a moving mass. However, (122) implies that for the upper simply supported beam acted upon by a moving mass we have

$$
\begin{gathered}
\omega_{q s s}\left[1+\frac{1}{1+T_{\mathrm{ral}} R_{I}\left(q^{2} \pi^{2} / L^{2}\right)}\right. \\
\left.-\frac{2 \gamma\left(\omega_{q s s}^{2}+\left(v^{2} q^{2} \pi^{2} / L^{2}\right)\right)}{\omega_{q s s}^{2}\left(1+T_{\mathrm{ral}} R_{I}\left(q^{2} \pi^{2} / L^{2}\right)\right)}\right]=\sigma_{q s s} ;
\end{gathered}
$$

that is,

$$
\frac{\omega_{q s s}\left(1+T_{\mathrm{ra} 1} R_{I}\left(q^{2} \pi^{2} / L^{2}\right)-\gamma\right)}{\left(1+T_{\mathrm{ra} 1} R_{I}\left(q^{2} \pi^{2} / L^{2}\right)+\gamma\left(v q \pi / \omega_{q s s} L\right)\right)}=\sigma_{q s s} .
$$

Evidently, from (124) and (125), the critical speed for the system of double-Rayleigh beam traversed by a moving mass is smaller than that of the same system under a moving force. This deduction implies that the response of the moving force as an approximation for the response of the moving mass can be not only misleading, but tragic.

An analysis similar to the above in the section for the particular series solutions of (76), (78), (79), (120), and (121) can also be carried out.

\section{Numerical Experiment and Discussion}

In this section, the coupling and individual effects of the moving mass and other dynamic variables such as viscoelastic parameter $\left(\varepsilon_{0}\right)$ of the layer, rotatory inertia correction factor $\left(R_{I}\right)$, velocity of the moving load $(v)$, and the layer stiffness $(k)$ on the dynamic response of a double-Rayleigh beam system traversed by a moving mass are examined and discussed for some classical boundary conditions.

In particular, the analysis carried out in the previous sections of this paper are numerically illustrated in this section. In the first part of this numerical experiment and for the purpose of numerical validation, the proposed solution procedure is initially applied to evaluate the dynamic response of an undamped simply supported double-beam system. To this end and since references for the analysis of double-Rayleigh beam system with linear viscoelastic Winkler type layer under a moving mass are scarce, the results are compared with those obtained in [25] where the dynamic response of a simply supported double-Euler-Bernoulli beam system traversed by a moving force had been studied. Thus, the effects of rotatory inertia and that of load mass are neglected in the present analysis (by setting $T_{\text {ral }}=0$ and $T_{\text {ra3 }}=0$ ). Following [25], the nondimensional deflections under moving force for the upper $W_{1 s s f}(L / 2, s) / v_{0}$ and lower $W_{2 s s f}(L / 2, s) / v_{0}$ Euler-Bernoulli beams are analysed. The static deflection at mid-span of a simply supported EulerBernoulli beam acted upon by a static force $P$ at point $x=L / 2$ which is denoted by $v_{0}$ and defined as (see Frýba (1972))

$$
v_{0}=\frac{2 P L^{3}}{\pi^{4} E I}
$$




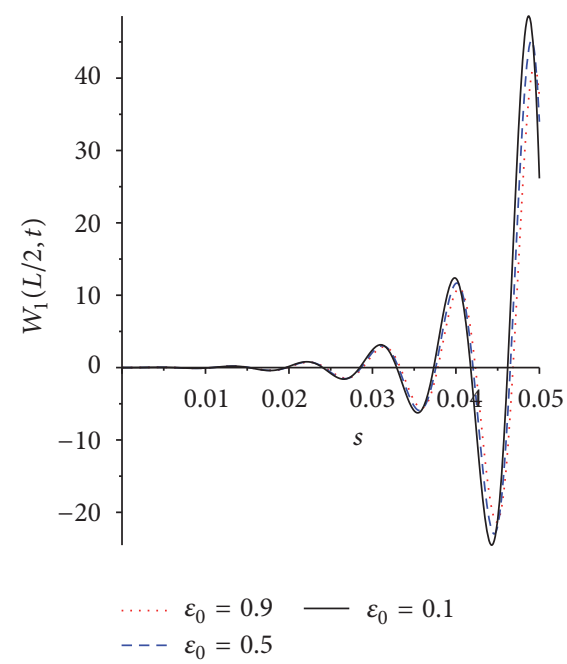

(a)

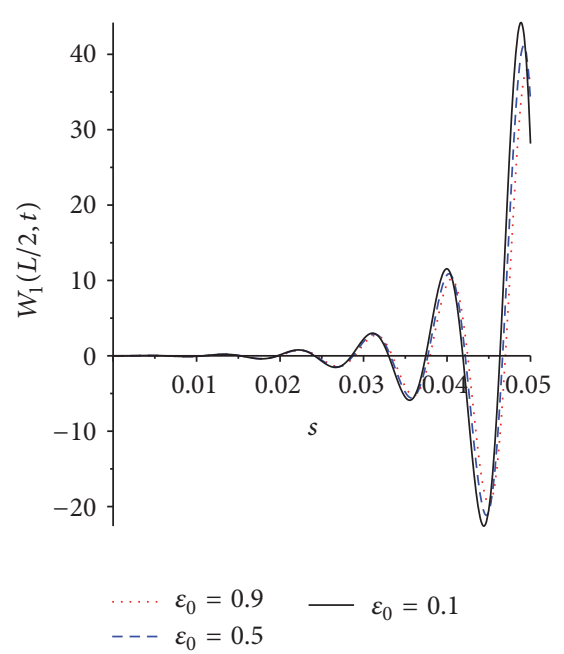

(b)

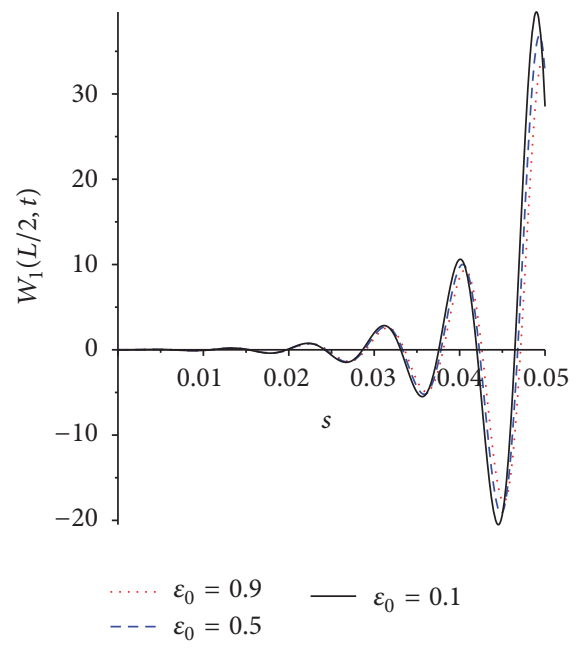

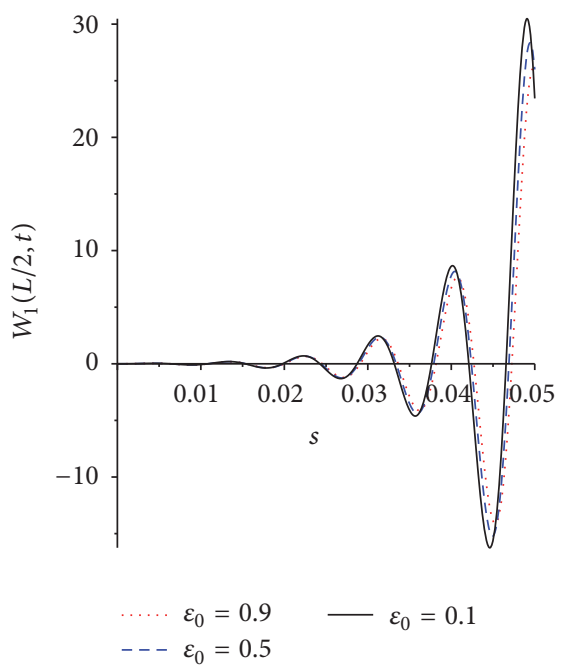

(d)

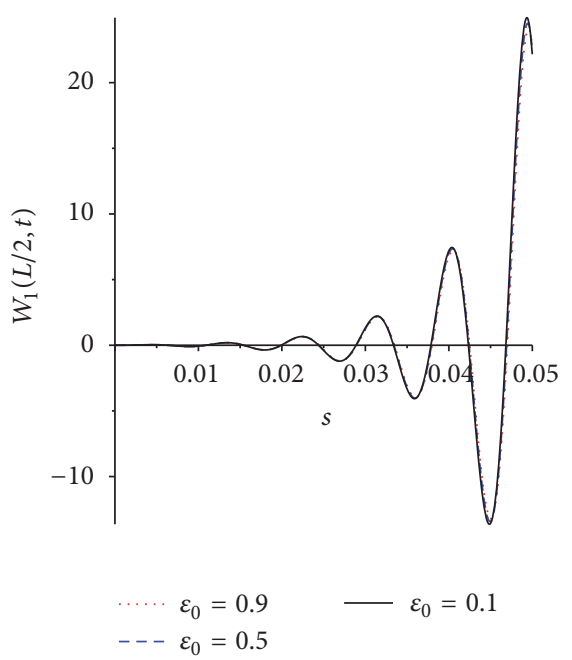

(e)

(c)

FIgUre 2: Continued. 


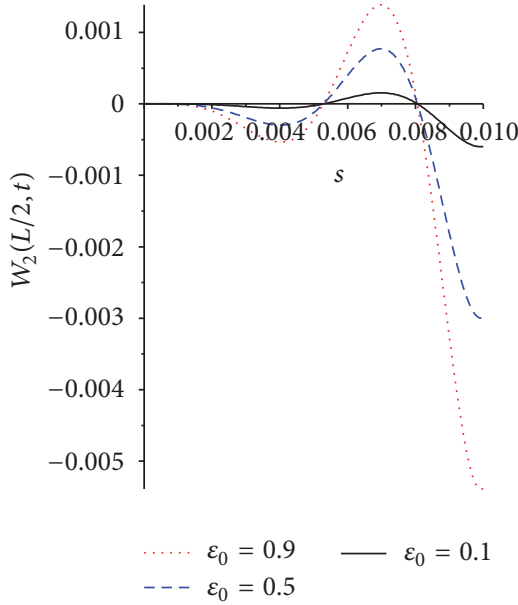

(f)

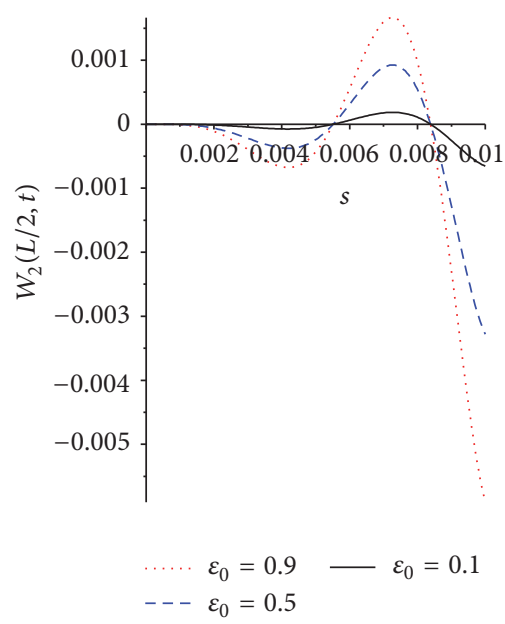

(g)

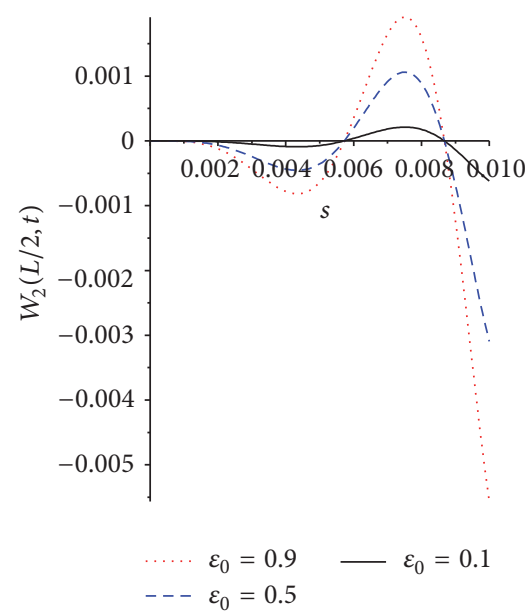

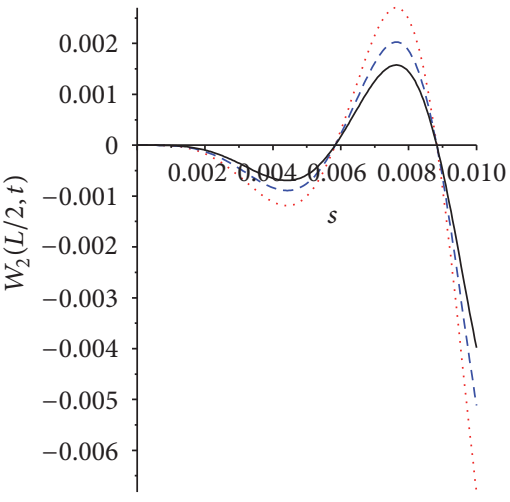

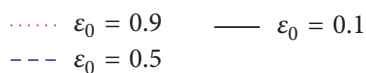

(i)

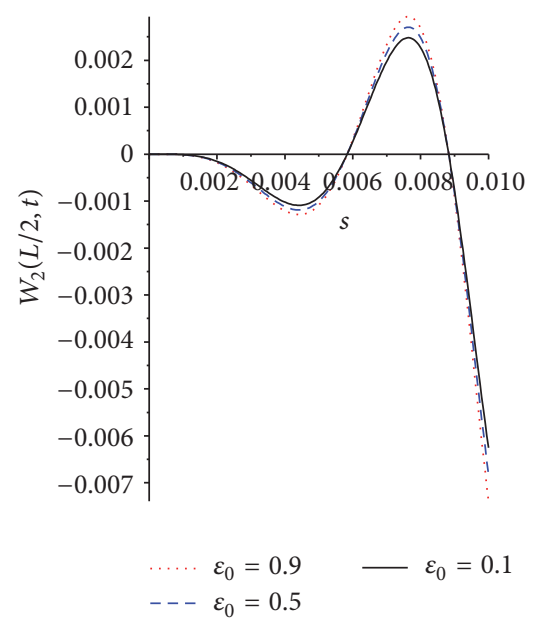

(j)

(h)

Figure 2: (a-e) The transverse dimensionless displacement responses for the simply supported upper Rayleigh beam $\left(W_{1}(L / 2, S)\right)$ for a velocity parameter $\alpha_{1}=0.1,(\mathrm{a}, \mathrm{f}) S_{1}=0.1,(\mathrm{~b}, \mathrm{~g}) S_{1}=1,(\mathrm{c}, \mathrm{h}) S_{1}=10,(\mathrm{~d}, \mathrm{i}) S_{1}=100$, and (e, j) $S_{1}=10000$. (f-j) The transverse dimensionless displacement responses for the simply supported lower Rayleigh beam $\left(W_{2}(L / 2, S)\right)$ for a velocity parameter $\alpha_{1}=0.1,(\mathrm{a}, \mathrm{f})$ $S_{1}=0.1,(\mathrm{~b}, \mathrm{~g}) S_{1}=1,(\mathrm{c}, \mathrm{h}) S_{1}=10,(\mathrm{~d}, \mathrm{i}) S_{1}=100$, and $(\mathrm{e}, \mathrm{j}) S_{1}=10000$. 


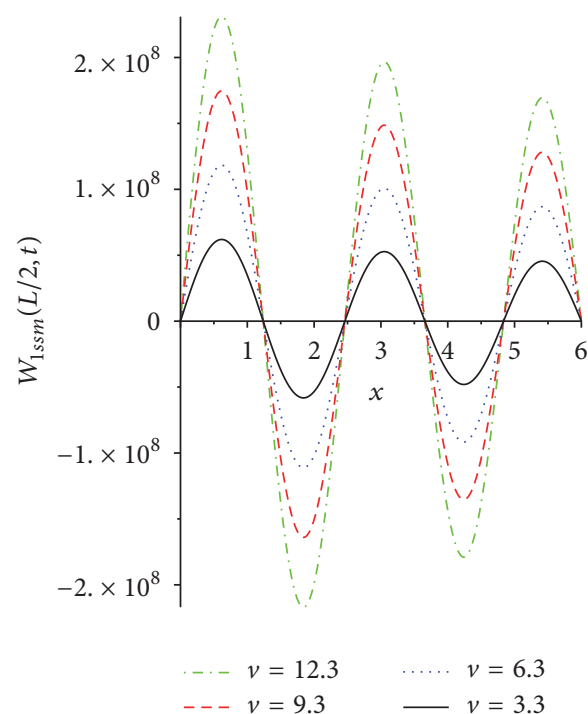

(i)

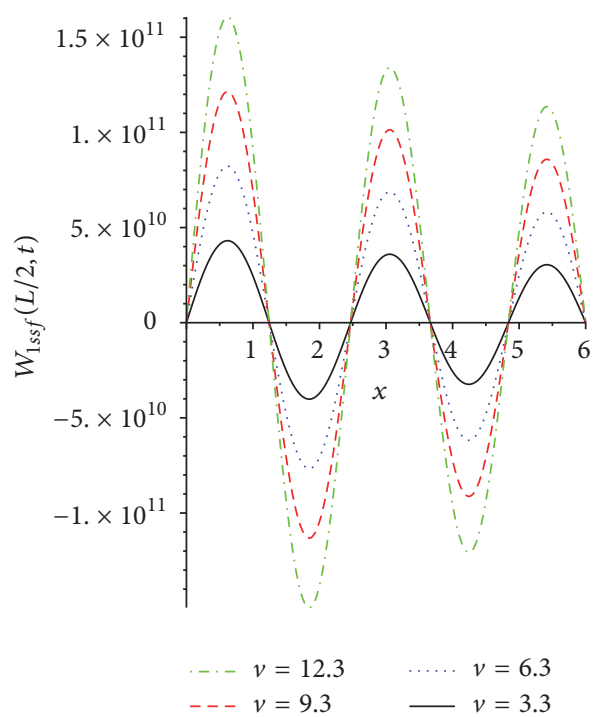

(ii)

(a)

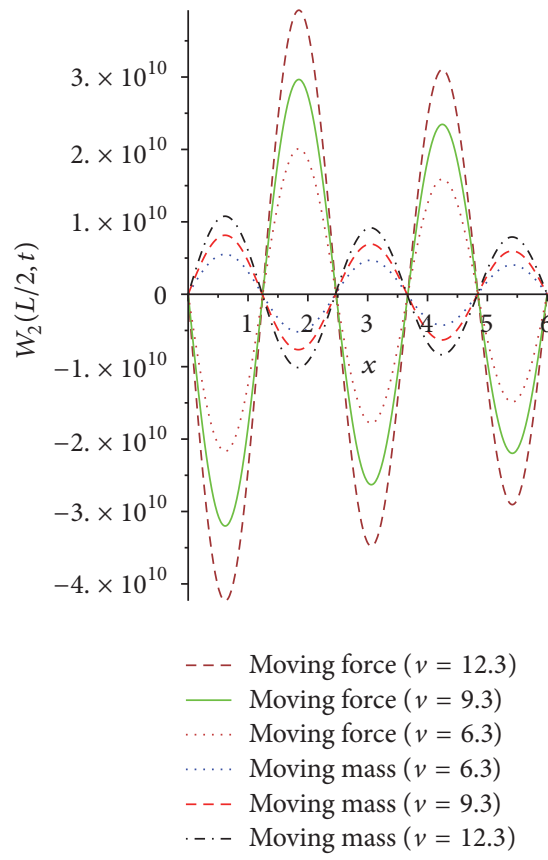

(b)

FIGURE 3: (a) The variation of velocity of the load on the transverse deflection of the upper Rayleigh beam due to moving mass and moving force, respectively. (b) The variation of velocity of the moving load on the transverse deflection of the lower Rayleigh beam due to moving mass and moving force.

is used in nondimensionalizing the deflections $\left(W_{i s s f} ; i=\right.$ $1,2)$. Also, the nondimensional time $s$ is defined as

$$
s=\frac{v t}{L}
$$

while the nondimensional velocity parameter $\alpha_{1}$ and critical speed $\alpha_{1 \mathrm{cr}}$ are defined as

$$
\alpha_{1}=\frac{v}{\alpha_{1 \mathrm{cr}}}
$$

$$
\alpha_{1 \mathrm{cr}}=\frac{\pi}{L}\left(\frac{E I}{\mu}\right)^{1 / 2}
$$

respectively. Also the nondimensional stiffness parameter of the Winkler type layer is given as

$$
s_{1}=\frac{k L^{4}}{E I} .
$$




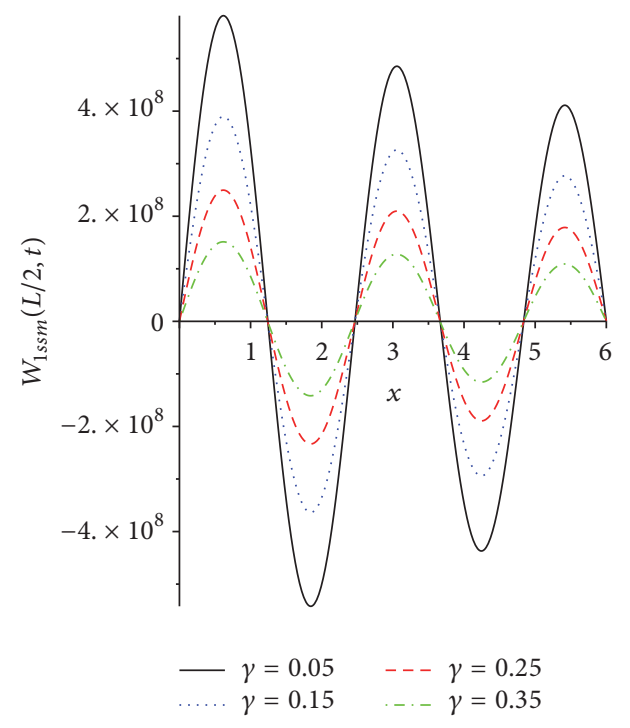

(a)

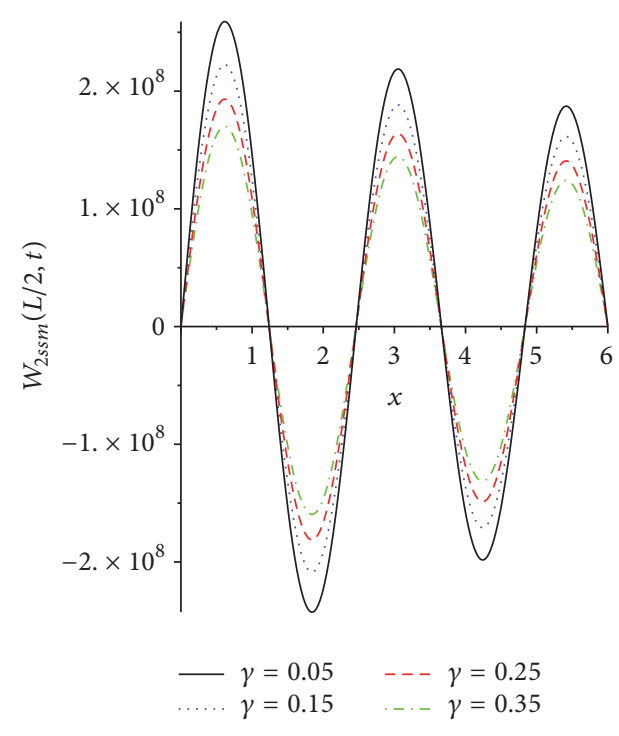

(b)

Figure 4: (a) The variation of mass ratio of the moving load on the transverse deflection of the upper Rayleigh beam. (b) The variation of mass ratio of the moving load on the transverse deflection of the lower Rayleigh beam.

The parameters selected for this first part of the analysis correspond to those used in the work in [25]. The transverse dimensionless displacements $\left(W_{i s s f}(L / 2, s), i=1,2\right)$ versus dimensionless time of a simply supported double EulerBernoulli beam system due to a moving force for various values of the dimensionless stiffness parameter $\left(s_{1}\right)$, viscoelastic parameter $\left(\varepsilon_{0}\right)$, and a fixed value of the velocity parameter $\left(\alpha_{1}=0.1\right)$ are displayed in Figures $2(\mathrm{a})-2(\mathrm{j})$. The first five graphs, Figures 2(a)-2(e), are for the upper beam while the remaining five graphs, Figures $2(f)-2(j)$, are for the lower beam. Figures 2(a)-2(e) depict dimensionless displacement response $W_{1 s s f}(L / 2, s)$ profile of the upper beam for variations in viscoelastic parameter $\left(\varepsilon_{0}\right)$. The results show that, depending on the nondimensional time $(s)$, an increase in the values of the viscoelastic parameter $\varepsilon_{0}$ leads to an increase or a decrease in the nondimensional displacement response, $W_{1 s s f}(L / 2, s)$, of the upper beam. Also, it is seen from these figures that the nondimensionless displacement response of the upper beam, $W_{1 s s f}(L / 2, s)$, decreases with increase in values of the stiffness parameter $\left(s_{1}\right)$, while the influence of the viscoelastic parameter $\left(\varepsilon_{0}\right)$ on $W_{1 s s f}(L / 2, s)$ reduces with increasing value of $\left(\varepsilon_{0}\right)$.

Also, values of $s_{1}$ smaller than 0.1 or higher than 10000 have irrelevant influence on the dimensionless displacement response $W_{1 s s f}(L / 2, s)$. It is, however, remarked at this juncture that if the viscoelastic parameter $\left(\varepsilon_{0}\right)$ is neglected, then very small value of the stiffness parameter $\left(s_{1}\right)$ amounts to dealing with weak elastically coupled beam system. Also, increasing the values $\left(s_{1}\right)$ means that the coupling between the two beams increases while a rigid coupling of the two beams is obtained for very high values of $\left(s_{1}\right)$. It is further observed that the values of $W_{1 s s f}(L / 2, s)$ involving rigid coupling (Figure $2(\mathrm{e})$ ) are reduced by $50 \%$ when compared to the corresponding ones involving weak elastic coupling
(Figure 2(a)). On the other hand, Figures 2(f)-2(j) show the nondimensional displacement responses $W_{2 s s f}(L / 2, s)$ for the lower Euler-Bernoulli beam having the velocity parameter $\left(\alpha_{1}=0.1\right)$ and various values of the viscoelastic parameter $\left(\varepsilon_{0}\right)$ and stiffness $\left(s_{1}\right)$. From these figures, it is found that an increase in the values of the stiffness parameter $\left(s_{1}\right)$ leads to an increase in the nondimensional displacement response, $W_{2 s s f}(L / 2, s)$, for the lower beam. It is remarked at this junction that all the above results for double finite Euler-Bernoulli beam system under moving force are in good agreement with those in [25] where a different approach is used.

In the second stage of this numerical experiment, the behaviour of a uniform double-Rayleigh beam system traversed by a moving mass or force and subjected to any of the classical end conditions is examined in order to obtain the effect of the load mass on such behaviour. Two vibrating configurations, namely, (i) simply supported double-Rayleigh beam system and (ii) clamped-clamped double-Rayleigh beam system, were considered. A computer program was developed on the basis of the abovementioned solution technique (discussed in Section 3). The computer program has been run for the following numerical data [24]: $L=6 \mathrm{~m}, E I=$ $16000 \mathrm{Nm}^{2}, k=10 \mathrm{~N} / \mathrm{m}^{2}, R_{I}=1,4,6,8, \mu=0.075 \mathrm{~kg} / \mathrm{m}^{-2}$, $v=3.3,6.3,9.3,12.3 \mathrm{~m} / \mathrm{s}^{2}, \varepsilon_{0}=0.1,0.2,0.3$, and 0.35 , and $\gamma_{1}=0.05,0.15,0.25$, and 0.35 . Without loss of generality it is assumed that the two beams are physically and geometrically identical.

6.1. Simply Supported Double-Rayleigh Beam System. For the first vibrating configuration, the transverse deflections $W_{i s s m}(x, t), i=1,2$, versus the load position $(x=v t)$ at some prescribed time $(t)$ of both the upper and lower beams of the simply supported uniform double-Rayleigh beams system 


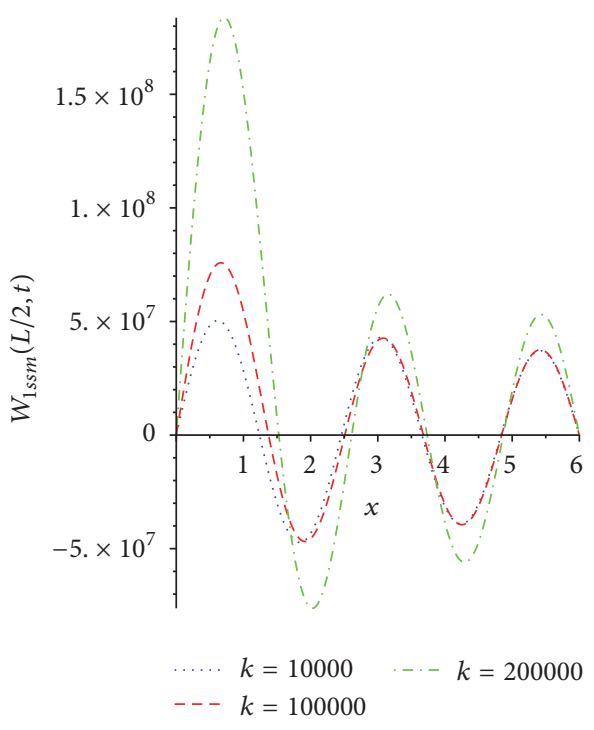

(i)

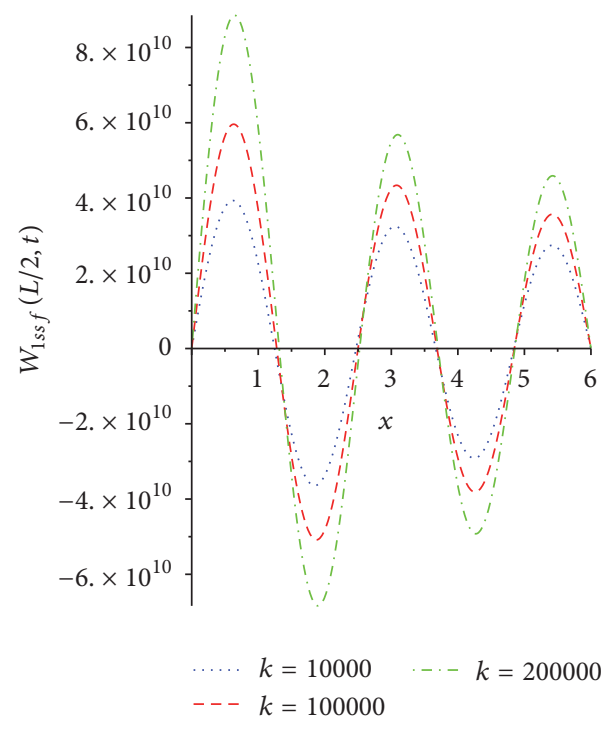

(ii)

(a)

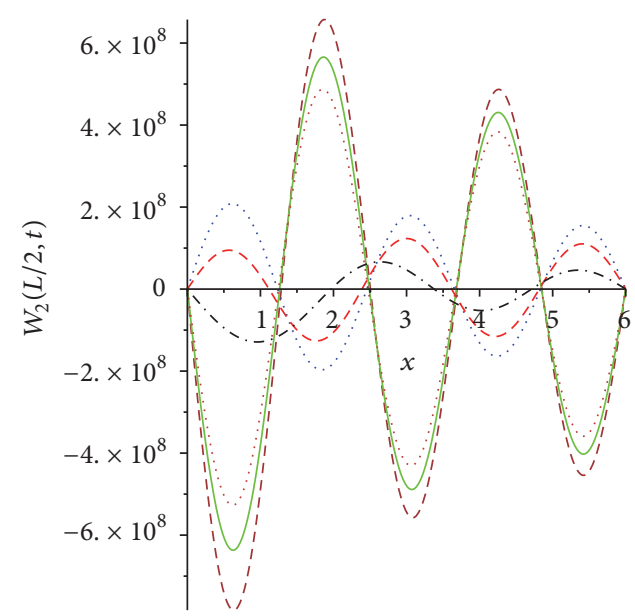

$$
\begin{aligned}
& \ldots \text { Moving force }(k=200000) \\
& \ldots \ldots \text { Moving force }(k=100000) \\
& \ldots \ldots \text { Moving force }(k=10000) \\
& \ldots \ldots \text { Moving mass }(k=200000) \\
& \text {-- } \text { Moving mass }(k=100000) \\
& \text {-.- Moving mass }(k=10000)
\end{aligned}
$$

(b)

Figure 5: (a) The variation of stiffness parameter of the layer on the transverse deflection of the upper Rayleigh beam due to moving mass and moving force, respectively. (b) The variation of stiffness parameter of the layer on the transverse deflection of the lower Rayleigh beam due to moving mass and moving force.

traversed by a load of mass ratio $\gamma_{1}$ moving at a constant velocity $(v)$ are considered.

In Figures 3(a)-7(a), the effects of variation of some governing variables parameters on the transverse deflection of the upper Rayleigh beam due to moving mass and moving force are presented, while the corresponding effects for the lower Rayleigh beam are displayed in Figures 3(b) $-7(b)$. In particular, Figure 3(a)(i) depicts the plot of variation in the velocity of a moving mass on the transverse deflection,
$W_{1 s s m}(x, t)$, of the simply supported upper Rayleigh beam due to the moving mass. It is seen from the plot that increase in the velocity leads to an increase in the response amplitude of the upper beam. The same trend is observed in Figure 3(a)(ii) which represents the behaviour of the upper Rayleigh beam acted upon by a moving force as opposed to a moving mass. Furthermore, Figures 3(a)(i) and 3(a)(ii) revealed that the response amplitude of the simply supported Rayleigh upper beam subjected to a moving force is greater than that of 


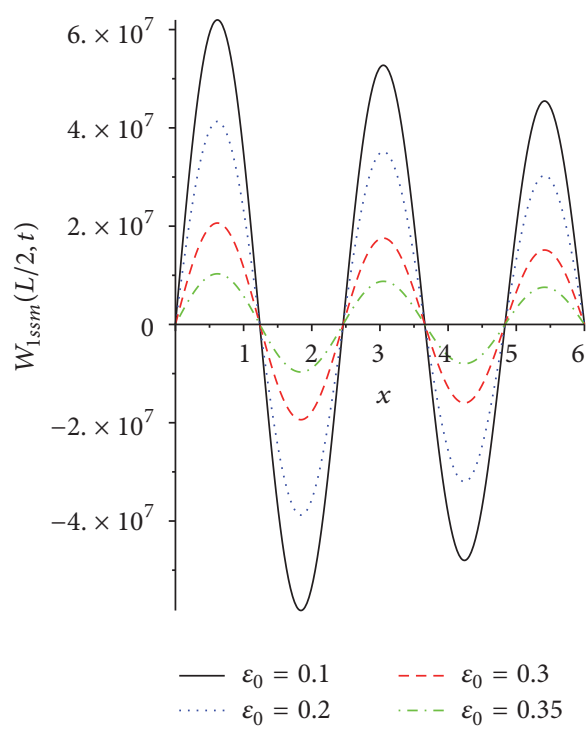

(i)

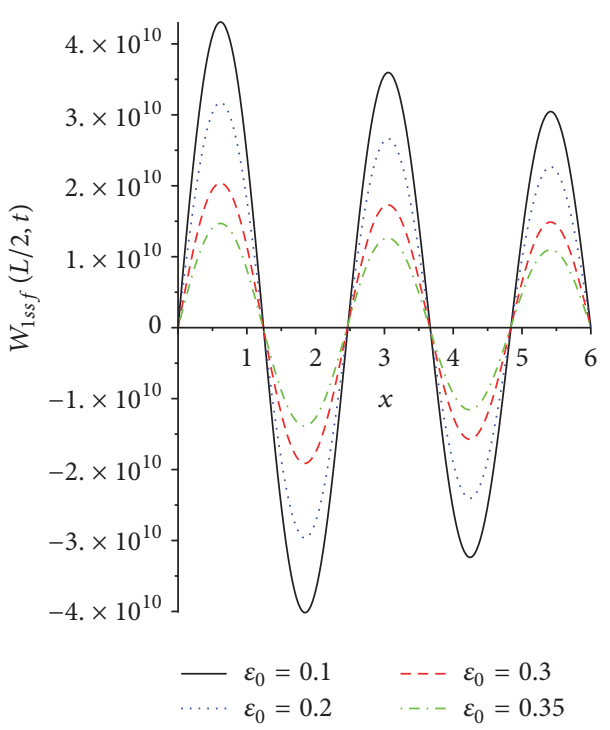

(ii)

(a)

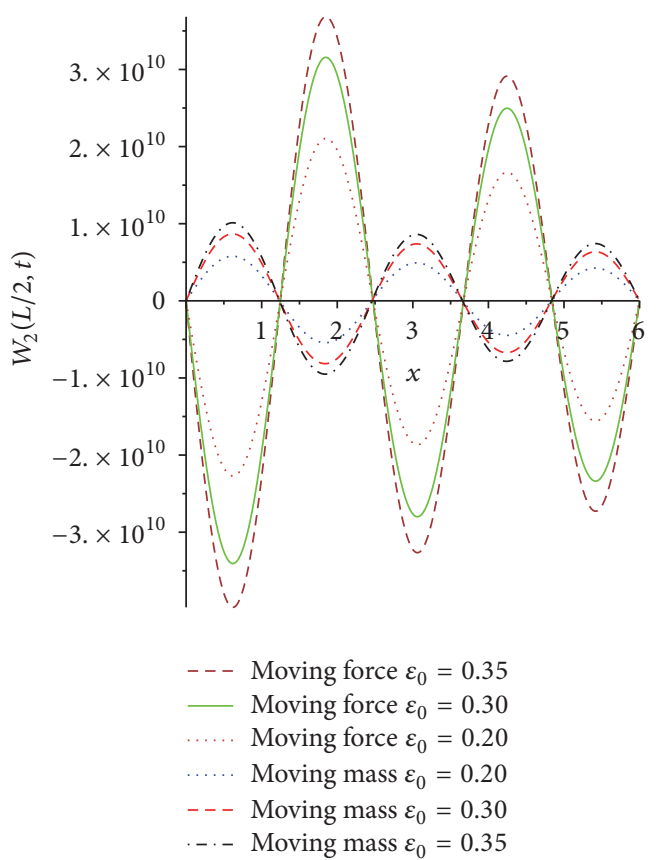

(b)

Figure 6: (a) The variation of viscoelastic parameter of the layer on the transverse deflection of the upper Rayleigh beam due to moving mass and moving force, respectively. (b) The variation of viscoelastic parameter of the layer on the transverse deflection of the lower Rayleigh beam due to moving mass and moving force.

the same beam under a moving mass thereby indicating the importance of the inclusion of the inertia effect of the moving load. Figure 4(a) represents the effect of mass ratio $\gamma_{1}=$ $\left(M_{L} / \mu L\right)$ on the transverse deflection of the upper simply supported Rayleigh beam. The plot indicates that increase in $\gamma_{1}$ decreases the response amplitude of the upper beam. The effect of the stiffness, $k$, of the viscoelastic Winkler type layer on the transverse displacement of the simply supported upper beam under a moving mass is presented in Figure 5(a). Increase in the value of the stiffness is found to cause an increase in the amplitude of the deflection of the upper beam. The same result is obtained when the same uniform simply supported upper beam is acted upon by a moving force as shown in Figure 5(a)(ii). However, Figures 5(a)(i) and 5(a)(ii) show that the response amplitude of the beam with a moving mass is smaller than that involving a moving 


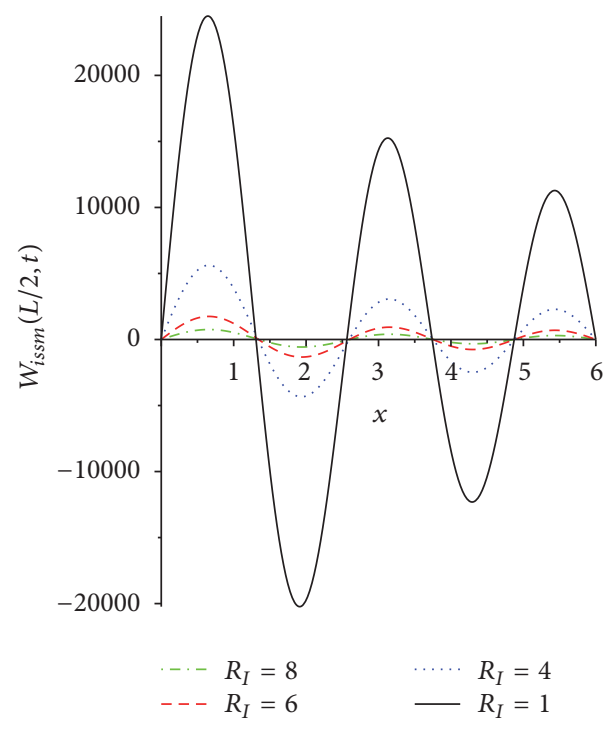

(i)

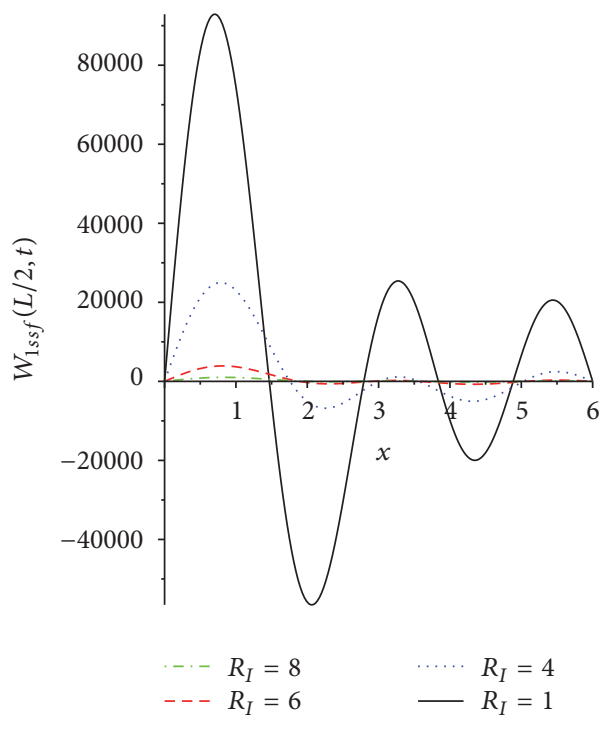

(ii)

(a)

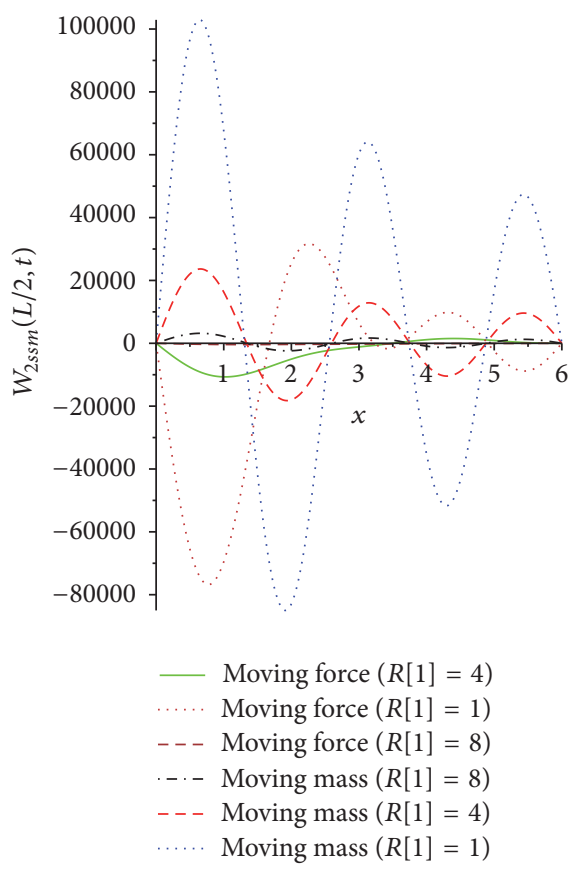

(b)

Figure 7: (a) The variation of rotatory inertia on the transverse deflection of the upper Rayleigh beam due to moving mass and moving force, respectively. (b) The variation of rotatory inertia on the transverse deflection of the lower Rayleigh beam due to moving mass and moving force.

force. The influence of the viscoelastic parameter $\left(\varepsilon_{0}\right)$ on the transverse displacement of the simply supported upper beam is presented in Figure 6(a). Figure 6(a)(i) depicts the graph of the effect of $\left(\varepsilon_{0}\right)$ on the transverse displacement involving a moving mass while the one involving a moving force is shown in Figure 6(a)(ii). It is found from the two figures that an increase in $\varepsilon_{0}$ leads to an increase in the response amplitude of the deflection of the upper beam. However the response amplitude of the deflection due to the moving mass is observed to be smaller than that due to a moving force. Figures 7(a)(i) and 7(a)(ii) illustrate the variation due to rotatory inertia correction factors $\left(R_{I}\right)$ on the transverse deflections of the simply supported upper Rayleigh beam due to either moving mass in Figure $7(\mathrm{a})(\mathrm{i})$ or moving force in Figure 7(a)(ii). An increase in $R_{I}$ leads to a decrease in the amplitude of the transverse deflections of the upper beam 


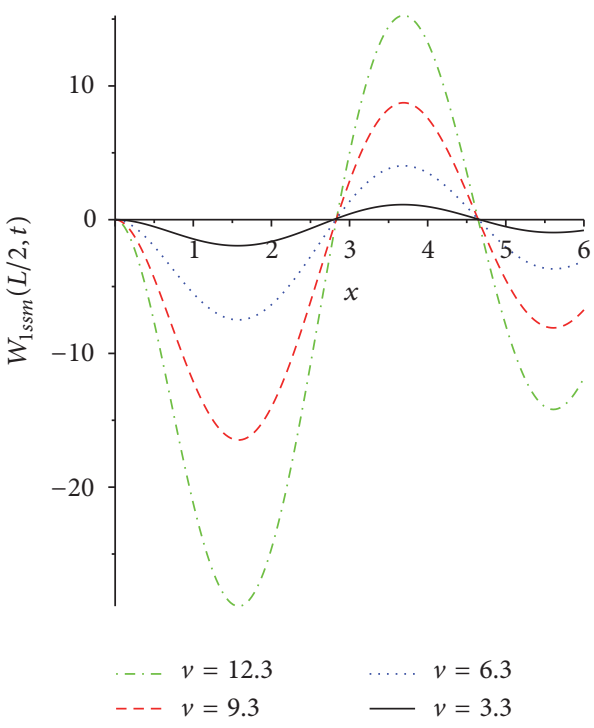

(i)

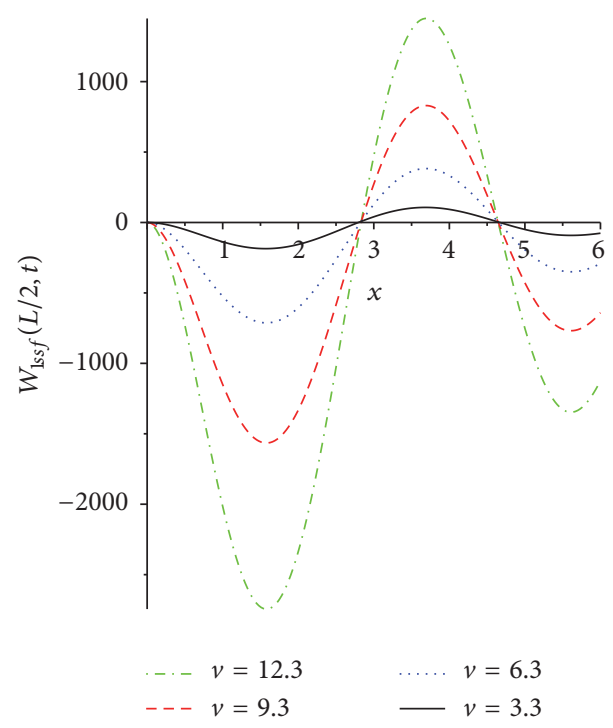

(ii)

(a)

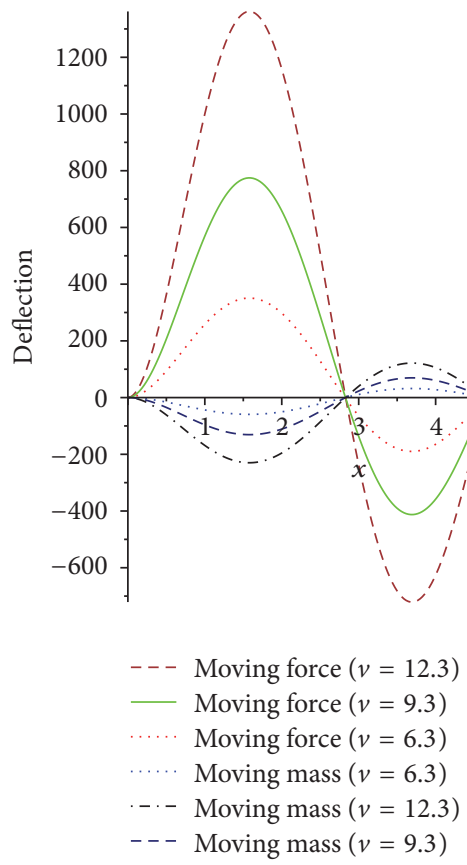

(b)

FIGURE 8: (a) The variation of the velocity of the load on the transverse deflection of the clamped-clamped upper Rayleigh beam due to moving mass and moving force, respectively. (b) The variation of the velocity of the load on the transverse deflection of the clamped-clamped lower Rayleigh beam due to moving mass and moving force.

for both moving mass and moving force such that amplitude of the deflection due to moving force is greater than the corresponding one under moving mass.

Now the behaviour of the lower Rayleigh beam is discussed as follows. Figure 3(b) depicts the comparison of the influence of velocity on the transverse deflections of the lower beam due to both moving force and moving mass, respectively. It is observed that the transverse deflection of the lower beam $W_{2 s s f}(x, t)$ due to moving force increases as velocity increases. Same trend holds for the moving mass case. Moreover, the response amplitudes of the transverse deflection due to the moving force are greater than those due to the moving mass. Figure 4(b) illustrates the variation in the mass ratio $\left(\gamma_{1}\right)$ on the transverse deflection of the simply supported lower beam. Clearly, an increase in $\left(\gamma_{1}\right)$ leads to a decrease in the response amplitude of the lower beam. The effects of the stiffness $(k)$ of the connecting viscoelastic layer on the transverse deflection of the lower simply supported 


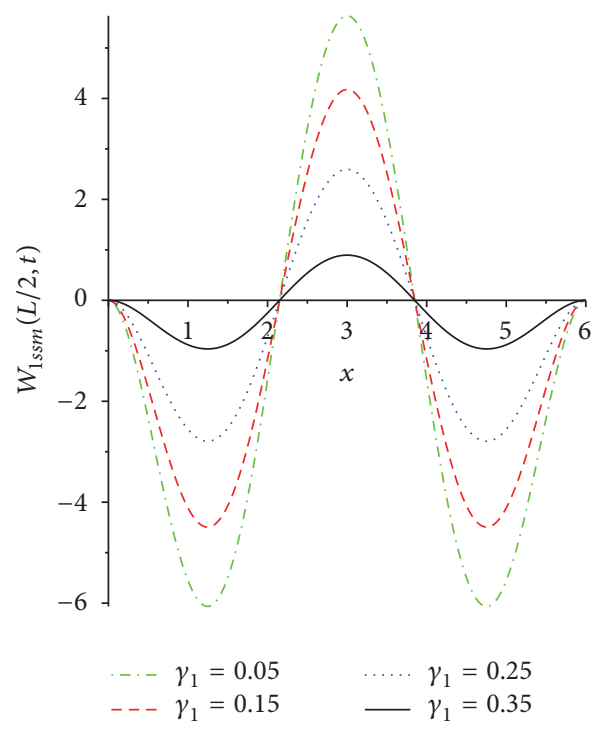

(a)

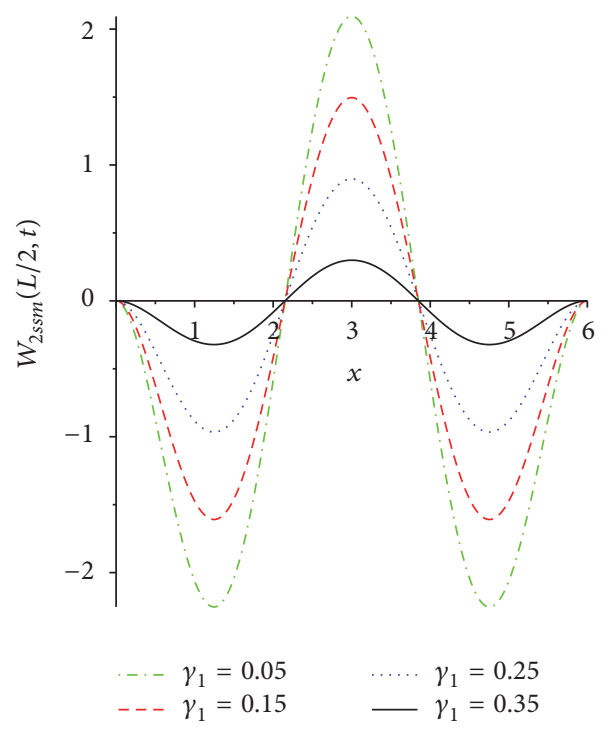

(b)

Figure 9: (a) The variation of mass ratio of the load on the transverse deflection of the clamped-clamped upper Rayleigh beam due to moving mass. (b) The variation of mass ratio of the load on the transverse deflection of the clamped-clamped lower Rayleigh beam due to moving mass.

beams for both moving mass and moving force are displayed in Figure 5(b). It is noticed that increase in $(k)$ decreases the response amplitude for the moving mass while the reverse is the case for the moving force. Figure 6(b) shows the comparison of the effect of the viscoelastic parameter $\left(\varepsilon_{0}\right)$ on the response deflection of the lower Rayleigh beam for both moving mass and moving force. It is found that, for both cases, increasing $\varepsilon_{0}$ yields an increase in the response amplitude of the simply supported lower Rayleigh beam. However, the response amplitude of the moving force is greater than that of the moving mass. The comparison of the influence of rotatory inertia correction factor $\left(R_{I}\right)$ on the transverse deflections of the lower Rayleigh beam for both moving mass and moving force is presented in Figure 7(b). It is observed that the response amplitude of deflections of the moving mass decreases as $R_{I}$ increases and the response amplitudes are greater than the corresponding ones observed for a moving force.

6.2. Clamped-Clamped Double-Rayleigh Beam System. The second vibrating configuration, that is, the behaviour of clamped-clamped double-Rayleigh beam system traversed by a moving mass, is numerically illustrated as follows: Figures 8(a)-12(a) show the deflections of the clamped-clamped upper Rayleigh beam when the clamped-clamped doubleRayleigh beam system is traversed by a moving mass or a moving force for various values of stiffness parameter $(k)$, viscoelastic parameter $\varepsilon_{0}$, mass ratio $\gamma_{1}$, rotatory inertia correction factor $\left(R_{I}\right)$, and velocity $(v)$.

Figures 8(a)(i) and 8(a)(ii) indicate the effect of velocity $(v)$ of the moving mass on the transverse deflection, $W_{1 c c f}(x, t)$, of the clamped-clamped upper Rayleigh beam. From the plots, an increase in the velocity $(v)$ is seen to cause an increase in the response amplitude of the beam. Similarly an increase in the velocity $(v)$ is shown in Figure 8(a)(ii) to increase the response amplitude of the same beam when the effect of the inertia of the moving load is neglected. It is, however, observable from Figures 8(a)(i) and 8(a)(ii) that the response amplitude due to the moving force is greater than that due to the moving mass. In Figure 9(a), the effect of the mass ratio $\gamma_{1}$ on the transverse deflections of the clamped-clamped upper beam traversed by a moving mass is illustrated. As seen from the plot, an increase in the mass ratio is observed to lower the response amplitudes of the beam. Figures 10(a)(i) and 10(a)(ii) depict the effect of stiffness of the viscoelastic layer $(k)$ on the response amplitude of the clamped-clamped upper beam due to moving mass and moving force, respectively. As seen from the plots, an increase in the stiffness is observed to enhance the response amplitude of the upper Rayleigh beam due to the moving mass. It is also noticed that same results are observed when the effect of the inertia of the moving load is neglected (see Figure 10(a)(ii)). Figures 11(a)(i) and 11(a)(ii) show the effect of viscoelastic parameter of the layer $\left(\varepsilon_{0}\right)$, on the response amplitude of the clamped-clamped upper beam under the moving mass and moving force, respectively. Moreover, as shown in both figures, as $\left(\varepsilon_{0}\right)$ increases, it is observed that the response amplitudes in the two cases increase. In Figures 12(a)(i) and 12(a)(ii) the effects of rotatory correction factor $\left(R_{I}\right)$ due to moving mass and moving force, respectively, are presented. From the plots, an increase in $R_{I}$ is observed to decrease the response amplitudes in each case. It is, however, important to remark at this juncture that, for Figures 10(a)(i), 10(a)(ii), 11(a)(i), 11(a)(ii), 12(a)(i), and 12(a)(ii), the response amplitudes of the clamped-clamped upper beam due to moving force are greater than the corresponding ones due to moving mass. 


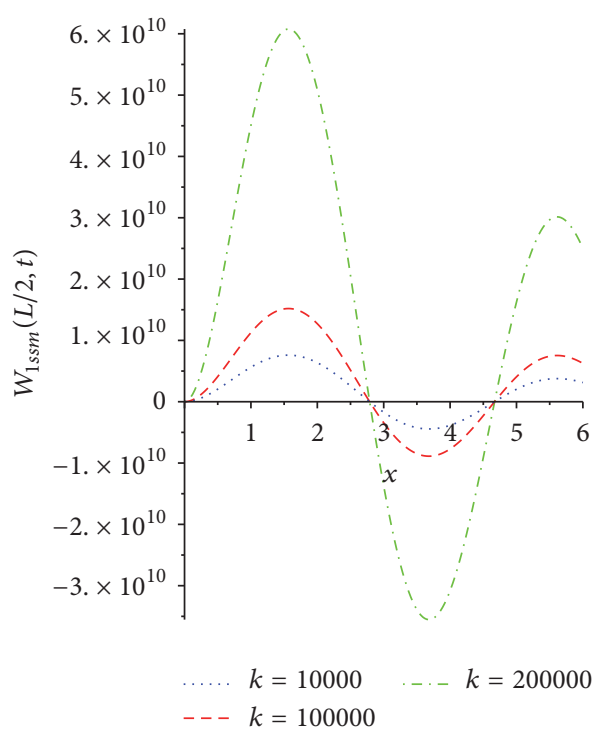

(i)

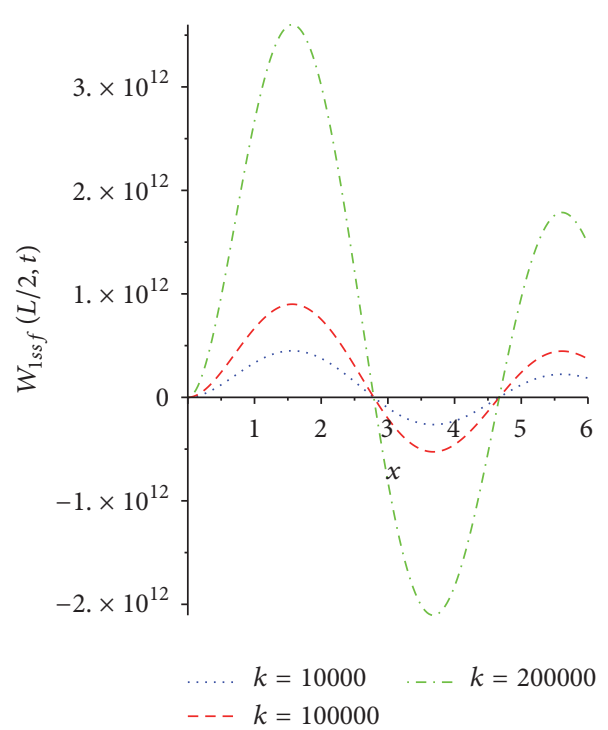

(ii)

(a)

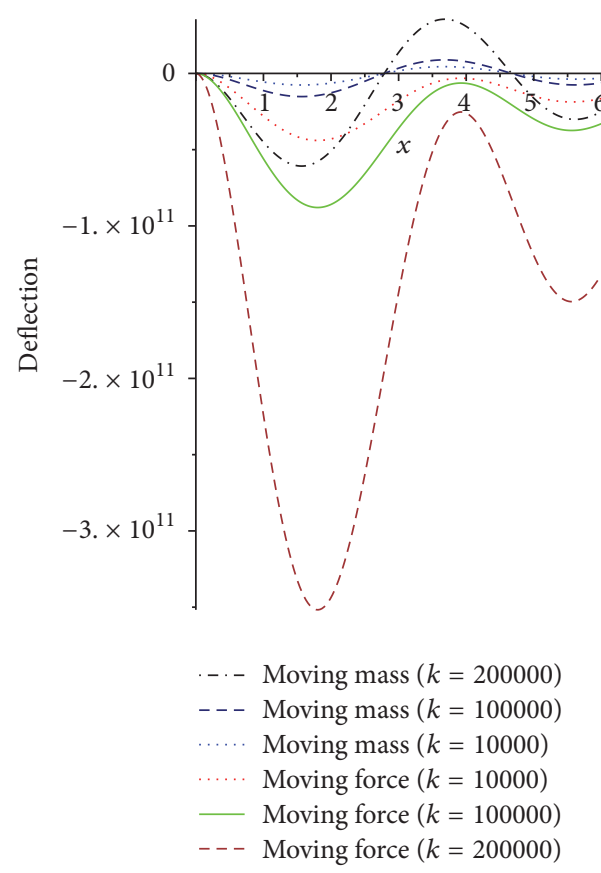

(b)

Figure 10: (a) The variation of stiffness of the layer on the transverse deflection of the clamped-clamped upper Rayleigh beam due to moving mass and moving force, respectively. (b) The variation of stiffness of the layer on the transverse deflection of the clamped-clamped lower Rayleigh beam due to moving mass and moving force.

Figure $8(\mathrm{~b})$ represents the response of the clampedclamped lower Rayleigh beam to variation of the velocity of the moving load when the load mass is retained or neglected. The figure shows that increasing the values of the speed parameter $(v)$ increases the response amplitudes due to both the moving force and moving mass cases. However, it is observable that a greater response amplitude is attained for the same value of $v$ in the case involving moving force when compared to the corresponding one due to moving mass.
Figure 9(b) presents the response of the clampedclamped lower Rayleigh beam due to variation of mass ratio $\left(\gamma_{1}\right)$ when load mass effect is considered. The plot indicates that as $\left(\gamma_{1}\right)$ increases, response amplitude of deflections decreases.

Figure 10(b) shows the response observed on the lower clamped-clamped Rayleigh beams for the cases when the load mass is either retained or neglected for variation of stiffness parameter $(k)$. It is noticeable from the figure that 


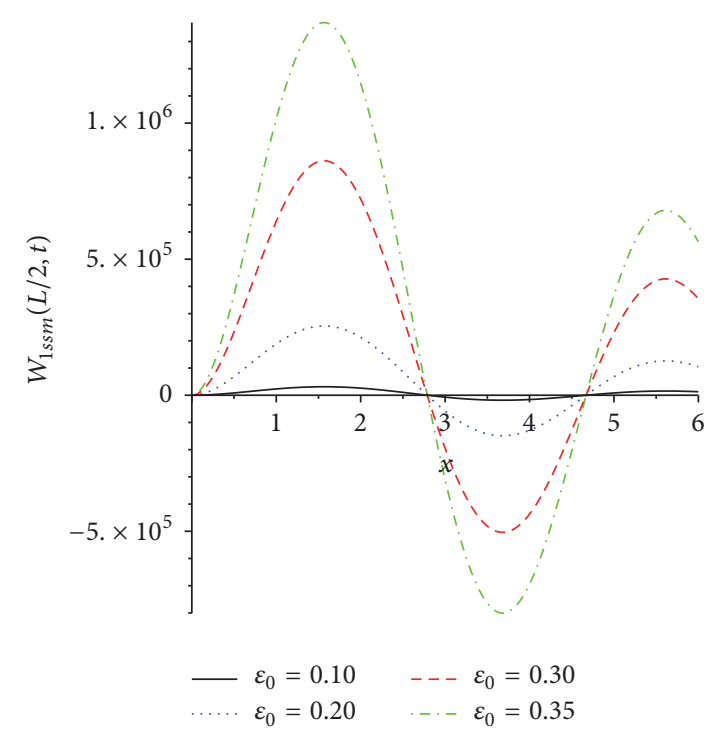

(i)

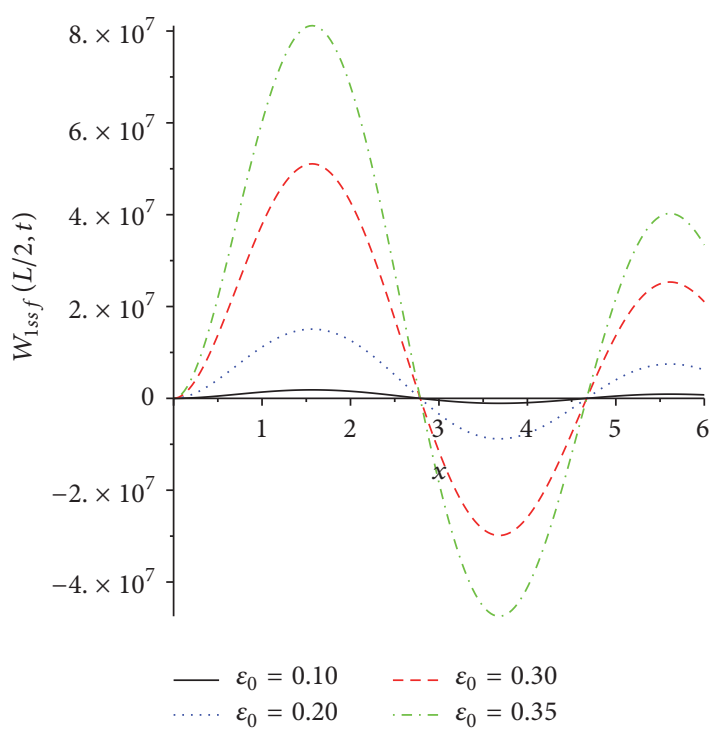

(ii)

(a)

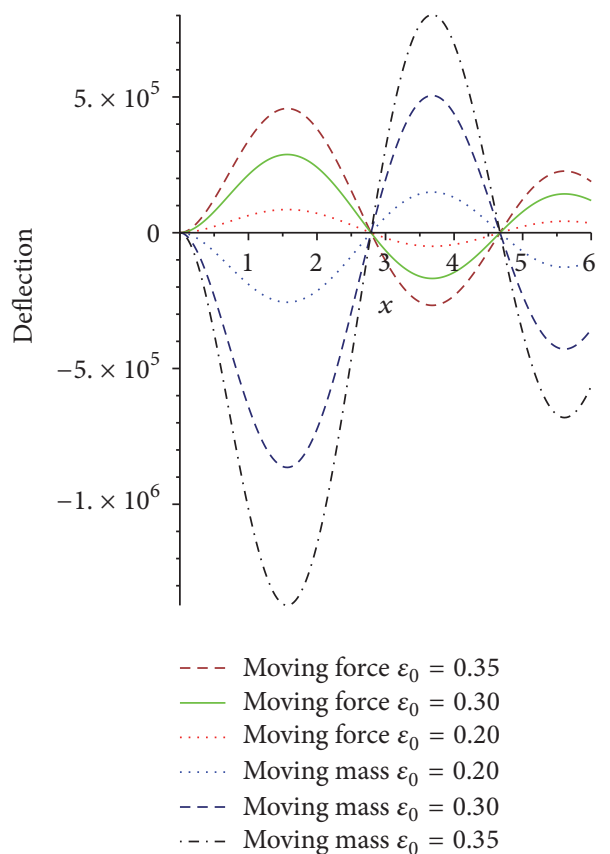

(b)

FIGURE 11: (a) The variation of viscoelastic parameter of the layer on the transverse deflection of the clamped-clamped upper Rayleigh beam due to moving mass and moving force, respectively. (b) The variation of viscoelastic parameter of the layer on the transverse deflection of the clamped-clamped lower Rayleigh beam due to moving mass and moving force.

increasing the stiffness parameter $(k)$ leads to an increase in the response amplitude for the case when the moving mass is retained while the same trend is observed for the case when the moving mass is neglected.

Figure 11(b) presents the effect of moving mass and moving force on the response of the clamped-clamped lower Rayleigh beam for various values of viscoelastic parameter $\left(\varepsilon_{0}\right)$. The plot for the moving mass and moving force indicates that increasing $\left(\varepsilon_{0}\right)$ leads to an increase in response amplitudes of the deflections of the beam. However, these responses are greater in the case involving moving mass when compared to the corresponding ones not involving moving mass.

In Figure 12(b), the effects of variation of rotatory inertia correction factor $\left(R_{I}\right)$ on the responses of clamped-clamped lower Rayleigh beam for both the moving mass and moving force are presented. It is observable that increasing $R_{I}$ in the cases involving moving mass and moving force leads to a 


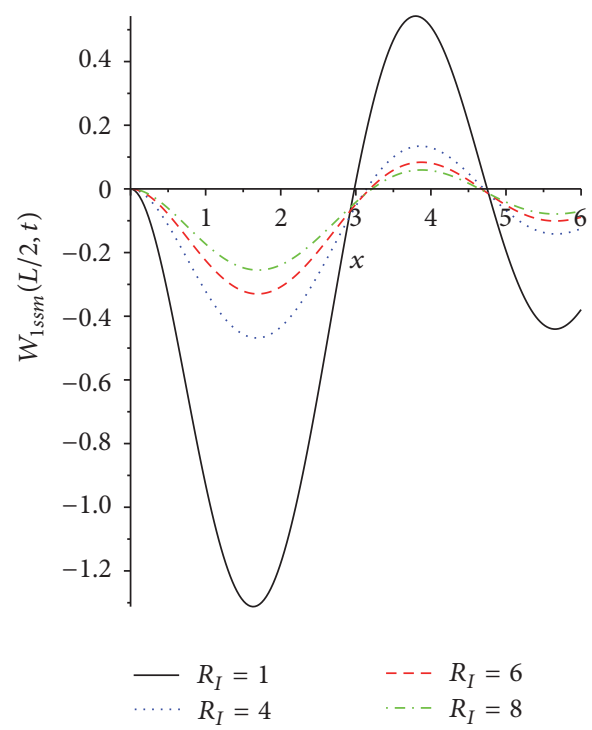

(i)

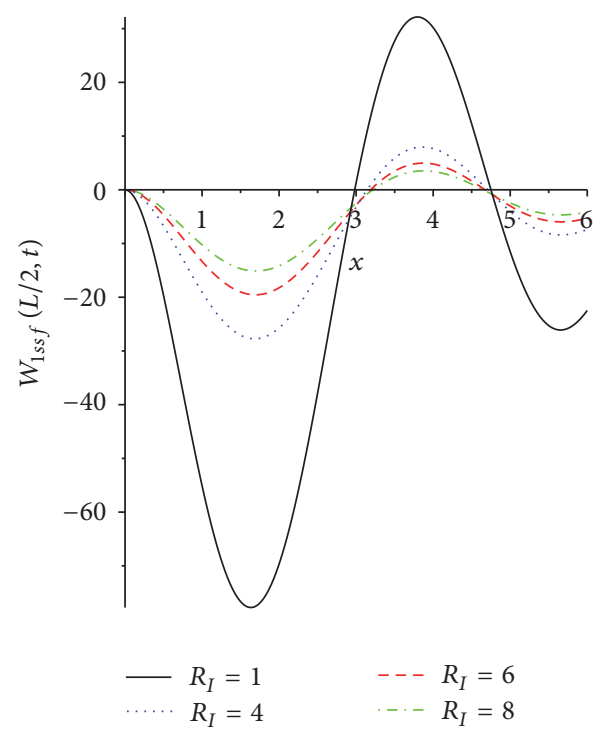

(ii)

(a)

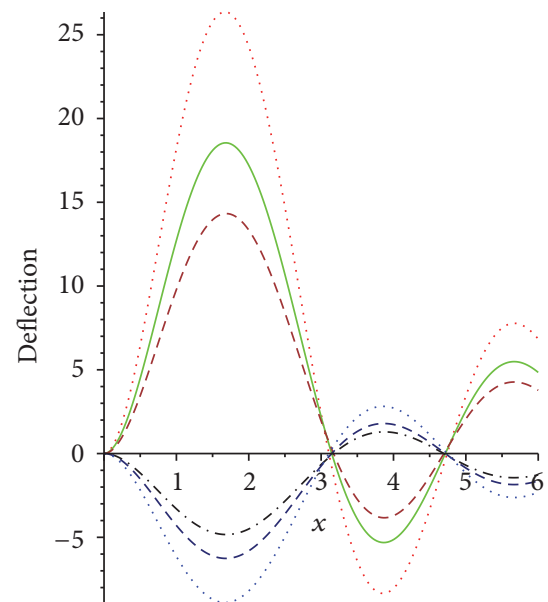

$$
\begin{array}{ll}
\cdots \cdots & \text { Moving force } R_{I}=4 \\
- & \text { Moving force } R_{I}=6 \\
--- & \text { Moving force } R_{I}=8 \\
-\cdots & \text { Moving mass } R_{I}=8 \\
--- & \text { Moving mass } R_{I}=6 \\
\cdots \cdots & \text { Moving mass } R_{I}=4
\end{array}
$$

(b)

FIGURE 12: (a) The variation of rotatory inertia of the load on the transverse deflection of the clamped-clamped upper Rayleigh beam due to moving mass and moving force, respectively. (b) The variation of rotatory inertia of the load on the transverse deflection of the clampedclamped lower Rayleigh beam due to moving mass and moving force.

decrease in the response amplitude of deflection. However, the response amplitudes due to moving mass are smaller than those due to moving force.

\section{Conclusion}

In this paper, an investigation of the forced vibration of a system comprising two finite beams separated by a Winkler viscoelastic core under a moving load has been carried out. The investigation has been done within the scope of Rayleigh beam theory which takes into account the effects of rotatory inertia. The present analysis was in particular carried out for both gravity and inertia effects of the moving load. In order to solve the governing initial-boundary value problem, a versatile solution technique suitable for any of the classical boundary conditions was developed. The technique involved, firstly, reducing the two governing fourthorder coupled partial differential equations to a set of two 
second-order ordinary differential equations by means of the generalized finite integral transform, then simplifying the latter by applying Struble's asymptotic method, and then solving the simplified set of two coupled differential equations with the aid of the differential transform method (DTM). One of the limitations of the proposed method is that it is a small parameter method and it is also valid in small region. However, the convergence domain could be enlarged using "After-treatment Technique (AT)" [30]. Another limitation is the iteration of the associated variational equation [31]. It was found that the results of the present method are in excellent agreement with those obtained in the literature for the case involving a concentrated moving force and simply supported end conditions [25]. It is observed that increasing the rotatory inertia correction factor, $R_{I}$, for the clamped-clamped lower Rayleigh beam, in the cases involving moving mass and force, leads to a decrease in the response amplitude of deflection. However the response amplitudes due to moving mass are smaller than those due to moving force. For the effect of the mass ratio, $\gamma_{1}$, on the lateral deflections of the clampedclamped upper beam traversed by a moving mass, it is seen that an increase in $\gamma_{1}$ lowers the response amplitudes of the beam. It is also noticed that an increase in the stiffness, $k$, of the connecting viscoelastic layer involving the transverse deflection of the lower simply supported beams leads to a decrease in the response amplitude for the moving mass while the reverse is the case for the moving force. Finally, the influence of ignoring the inertia of the moving mass on the dynamic behaviour of the finite double-Rayleigh beam system is presented.

\section{Competing Interests}

The authors declare that there is no conflict of interests regarding the publication of this paper.

\section{References}

[1] J. A. Gbadeyan and S. T. Oni, "Dynamic behaviour of beams and rectangular plates under moving loads," Journal of Sound and Vibration, vol. 182, no. 5, pp. 677-695, 1995.

[2] L. Frýba, Vibration of solids and structures under moving loads, vol. 1 of Monographs and textbooks on mechanics solids and fluids / Mechanics of structural systems, Springer, Dordrecht, Netherlands, 1972.

[3] E. A. Andi and S. T. Oni, "Dynamic behaviour under moving distributed masses of nonuniform Rayleigh beam with general boundary conditions," Chinese Journal of Mathematics, vol. 2014, Article ID 565826, 13 pages, 2014.

[4] S. Sadiku and H. H. E. Leipholz, "On the dynamics of elastic systems with moving concentrated masses," Ingenieur-Archiv, vol. 57, no. 3, pp. 223-242, 1987.

[5] E. Esmailzadeh and M. Ghorashi, "Vibration analysis of beams traversed by uniform partially distributed moving masses," Journal of Sound and Vibration, vol. 184, no. 1, pp. 9-17, 1995.

[6] J. A. Gbadeyan and M. S. Dada, "On the dynamic response of beams subjected to non-uniform distributed moving masses," Far East Journal of Applied Mathematics, vol. 33, no. 1, pp. 4359, 2008.
[7] J. A. Gbadeyan, O. T. Olotu, and M. S. Dada, “The Combined effects of uniform partially distributed moving mass, coriolis and centripetal forces on the response of Euler Bernoulli beams," ICASTOR Journal of Mathematical Science, vol. 3, no. 1, pp. 29-56, 2011.

[8] M. S. Dada, “The effects of linearly varying distributed moving loads on beams with Winkler foundation," in Contemporary Problems in Mathematical Physics, J. Govaerts and M. Norbert Hounkonnou, Eds., vol. 5, pp. 136-141, 2008, http://www.cipma .net/IMG/pdf/copromaph5.pdf.

[9] A. S. Idowu and J. A. Gbadeyan, “The response of a prestressed Bernoulli beam carrying an added mass to a concentrated moving load," Computing, Information Systems, Development Informatics and Allied Research Journal, vol. 7, no. 1, pp. 11-18, 2016.

[10] S. Mackertich, "Moving load on a Timoshenko beam," Journal of the Acoustical Society of America, vol. 88, no. 2, pp. 1175-1178, 1990.

[11] S. Mackertich, "Response of a beam to a moving mass," Journal of the Acoustical Society of America, vol. 92, no. 3, pp. 1766-1769, 1992.

[12] J. A. Gbadeyan and M. S. Dada, "Dynamic response of a Mindlin elastic rectangular plate under a distributed moving mass," International Journal of Mechanical Sciences, vol. 48, no. 3, pp. 323-340, 2006.

[13] J. A. Gbadeyan and M. S. Dada, "A comparison of dynamic responses of three versions of moving load problem involving elastic rectangular plates," Journal of Vibration and Control, vol. 17, no. 6, pp. 903-915, 2011.

[14] M. A. Foda and Z. Abduljabbar, "A dynamic green function formulation for the response of a beam structure to a moving mass," Journal of Sound and Vibration, vol. 210, no. 3, pp. 295306, 1998.

[15] V. Stojanović and P. Kozić, "Forced transverse vibration of Rayleigh and Timoshenko double-beam system with effect of compressive axial load," International Journal of Mechanical Sciences, vol. 60, no. 1, pp. 59-71, 2012.

[16] A. Mirzabeigy, R. Madoliat, and M. Vahabi, "Free vibrtaion analysis of two parallel beams connected together through variable stiffness elastic layer with elastically retrained ends," in Advances in Structural Engineering, pp. 1-13, 2016.

[17] M. Dublin and H. R. Friedrich, "Force responses of two elastics beams interconnected by springs-damped system," Journal of the Aeronautical Sciences, vol. 23, no. 9, Article ID 824829, 1956.

[18] J. M. Seelig and W. H. Hoppmann, "Impact on an elastically connected double-beam system," Journal of Applied Mechanics, vol. 31, pp. 621-626, 1964.

[19] J. M. Seelig and W. H. Hoppmann II, "Normal Mode Vibrations of Systems of Elastically Connected Parallel Bars," The Journal of the Acoustical Society of America, vol. 36, no. 1, p. 93, 1964.

[20] P. G. Kessel, "Resonances excited in an elastically connected double-beam system by a cyclic moving load," The Journal of the Acoustical Society of America, vol. 40, no. 3, pp. 684-687, 1966.

[21] P. G. Kessel and T. F. Raske, "Dynamic response of an elastically connected double-beam system due to a cyclic moving load," Journal of Acoustical Society of America, vol. 49, pp. 371-373, 1971.

[22] J. A. Gbadeyan, F. A. Hammed, and E. O. Titiloye, "Dynamic behaviour of viscoelastically connected beams carrying uniform partially distributed moving force," Nigeria Journal of Pure and Applied Sciences, vol. 20, pp. 1891-1905, 2005. 
[23] M. Nasirshoaibi, N. Mohammadi, and M. Nasirshoaibi, "Forced transverse vibration of a closed double single-walled carbon nanotube system containing a fluid with effect of compressive axial load," Shock and Vibration, vol. 2015, Article ID 435284, 11 pages, 2015.

[24] J. A. Gbadeyan and O. O. Agboola, "Dynamic behaviour of a double Rayleigh beam-system due to uniform partially distributed moving load," Journal of Applied Sciences Research, vol. 8, no. 1, pp. 571-581, 2012.

[25] M. Abu-Hilal, "Dynamic response of a double Euler-Bernoulli beam due to a moving constant load," Journal of Sound and Vibration, vol. 297, no. 3-5, pp. 477-491, 2006.

[26] V. Stojanovic and N. Nedic, "Robust identification of OE model with constrained output using optimal input design," Journal of the Franklin Institute, vol. 353, no. 2, pp. 576-593, 2016.

[27] V. Stojanovic, N. Nedic, D. Prsic, and L. Dubonjic, "Optimal experiment design for identification of ARX models with constrained output in non-Gaussian noise," Applied Mathematical Modelling, vol. 40, no. 13-14, pp. 6676-6689, 2016.

[28] J. K. Zhou, Differential Transformation and Its Applications for Electrical Circuits, Huazhong University Press, Wuhan, China, 1986.

[29] I. H. Abdel-Halim Hassan and V. S. Erturk, "Solutions of different types of the linear and non-linear higher-order boundary value problems by differential transformation method," European Journal of Pure and Applied Mathematics, vol. 2, no. 3, pp. 426-447, 2009.

[30] M. El-Shahed, "Application of differential transform method to non-linear oscillatory systems," Communications in Nonlinear Science and Numerical Simulation, vol. 13, no. 8, pp. 1714-1720, 2008.

[31] A. H. Nayfeh, Perturbation methods, John Wiley, 1973. 


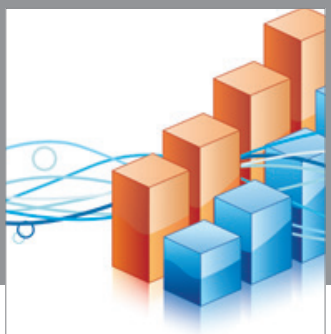

Advances in

Operations Research

vatem alat4

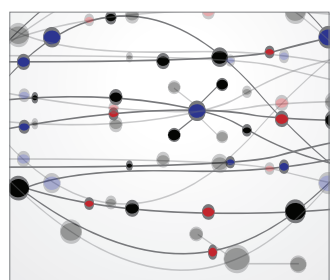

\section{The Scientific} World Journal
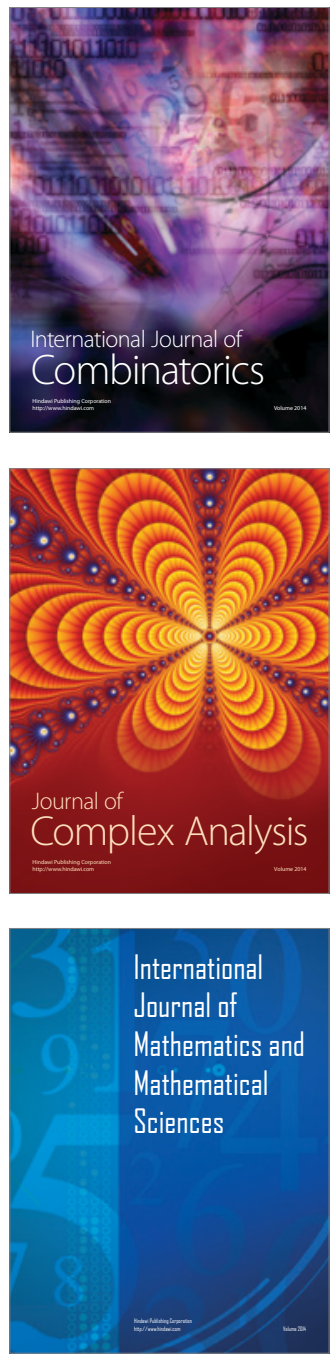
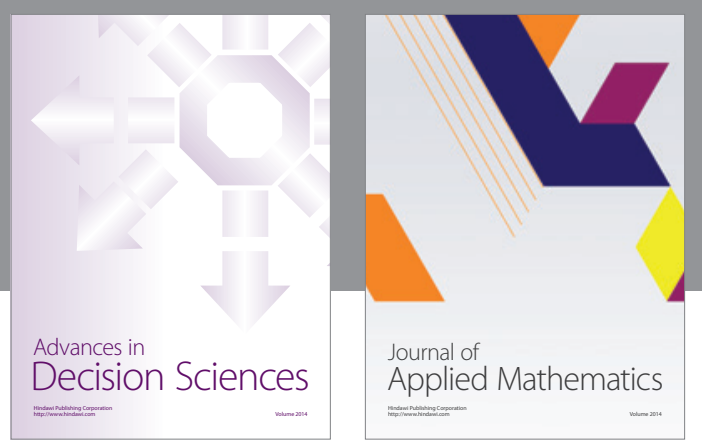

Algebra

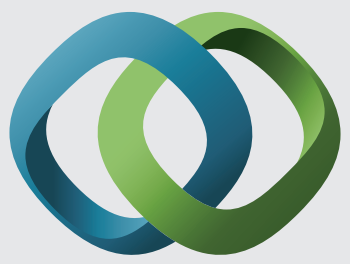

\section{Hindawi}

Submit your manuscripts at

https://www.hindawi.com
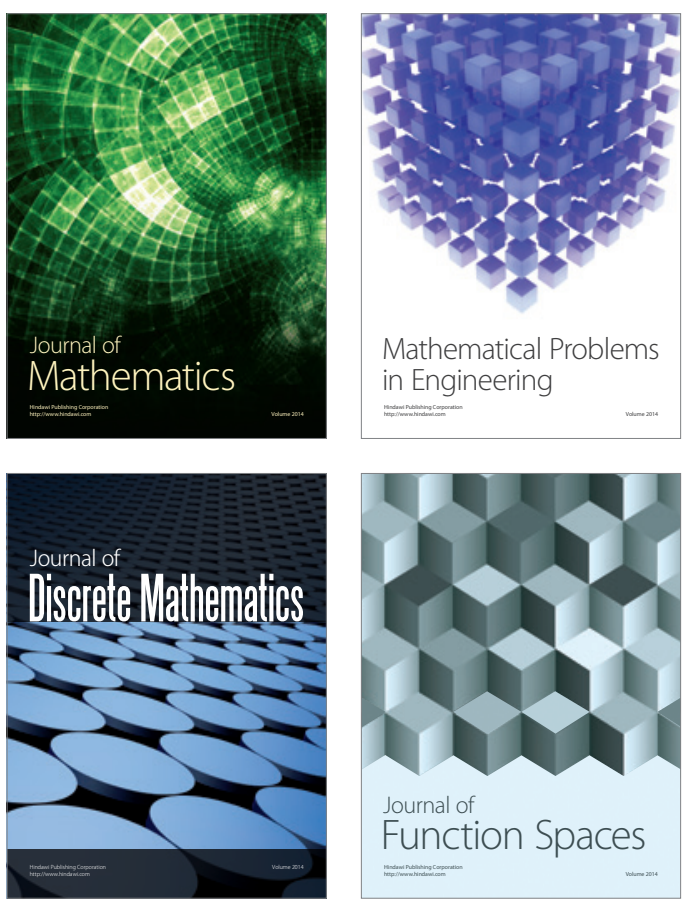

Mathematical Problems in Engineering
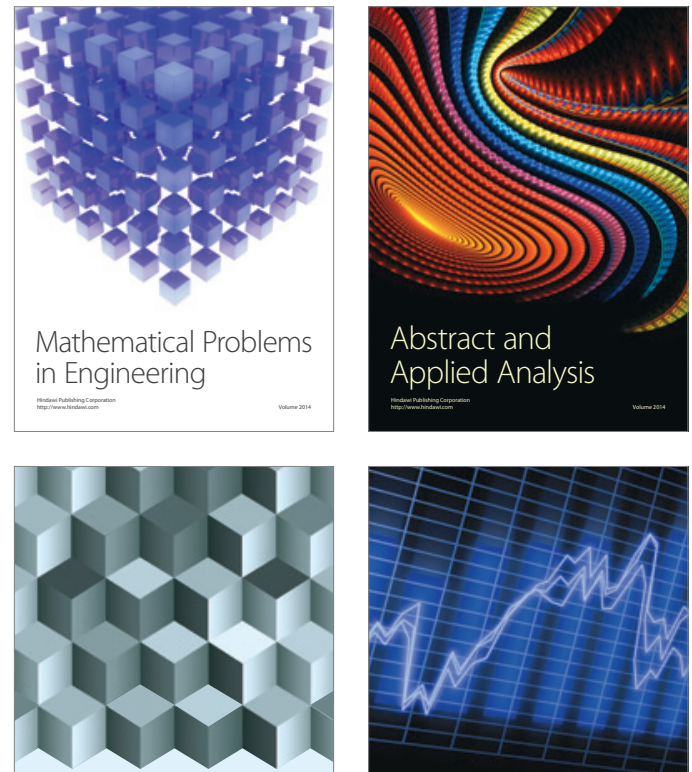

Journal of

Function Spaces

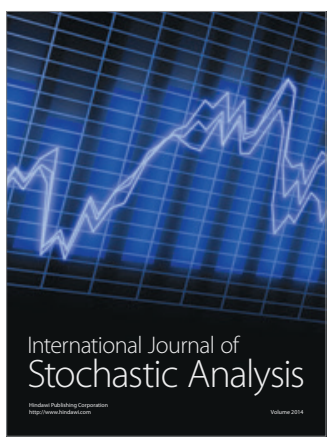

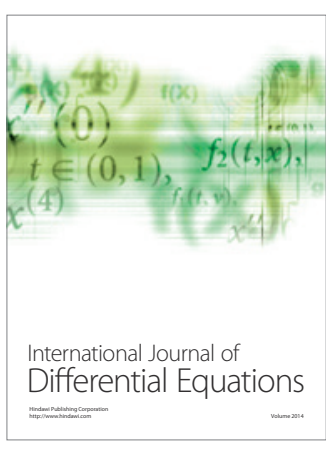
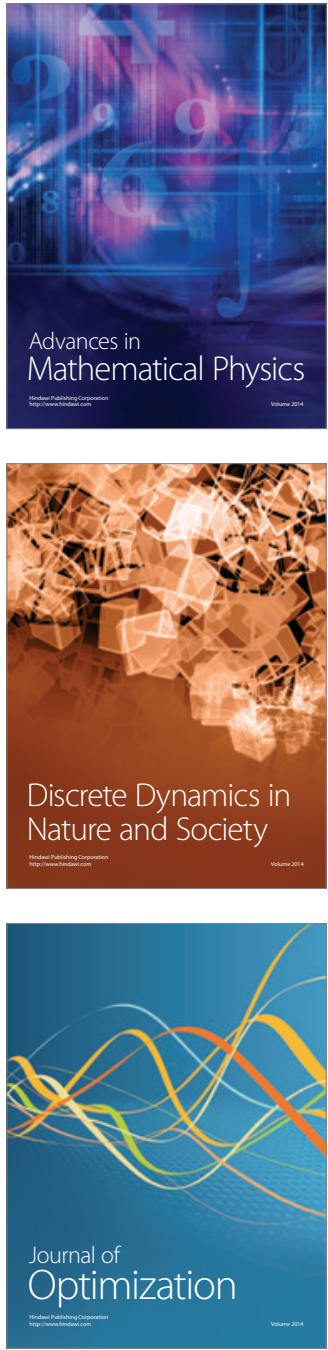